\title{
APPROXIMATE INERTIAL MANIFOLDS FOR NONLINEAR PARABOLIC EQUATIONS VIA STEADY-STATE DETERMINING MAPPING
}

\author{
YUNCHENG YOU \\ Department of mathematics \\ University of South Florida \\ Tampa, FL 33620, USA \\ (Received August 24, 1992 and in revised form December 1, 1993)
}

\begin{abstract}
For nonlinear parabolic evolution equations, it is proved that, under the assumptions of local Lipschitz continuity of nonlinearity and the dissipativity of semiflows, there exist approximate inertial manifolds (AIM) in the energy space and that the approximate inertial manifolds are constructed as the graph of the steady-state determining mapping based on the spectral decomposition. It is also shown that the thickness of the exponentially attracting neighborhood of the AIM converges to zero at a fractional power rate as the dimension of the AIM increases. Applications of the obtained results to Burgers' equation, higher dimensional reaction-diffusion equations, 2D Ginzburg-Landau equations, and axially symmetric Kuramoto-Sivashinsky equations in annular domains are included.
\end{abstract}

KEYWORDS. Approximate inertial manifold, absorbing property, steady-state determining mapping, exponential attraction, semilinear evolution equation, parabolic equation.

1991 AMS SUBJECT CLASSIFICATION CODES. 34C35, 35B40, 35K22, $35 \mathrm{Q} 10$.

\section{INTRODUCTION}

In the recent decade there have been rapidly expanding research progresses in the global dynamics (that is, the long time behavior of solutions) of nonlinear evolution equations with some sort of dissipativity. The existing theory consists of the existence of absorbing sets, the existence of a compact global attractor of finite Hausdorff and fractal dimensions for many typical parabolic and hyperbolic equations, the existence of inertial manifolds for some parabolic equations and nonlocal beam equations (mostly known only for the one space dimension cases), and the existence of approximate inertial manifolds by a variety of approaches. We refer to Hale [1] and Temam [2] as general references.

Consider a model equation, which represents different semilinear parabolic evolution equations under different assumptions, of the following form

$$
\begin{gathered}
\frac{d u}{d t}+A u+R(u)=f, \quad t \geq 0, \\
u(0)=u_{0} .
\end{gathered}
$$

Suppose that the global solution exists in a strong or weak sence in a Hilberet space $W$, and is denoted by $S(t) u_{0}=u\left(t, u_{0}\right), t \geq 0$, the nonlinear continuous mapping $S(t), t \geq 0$, is called the solution semigroup, or semiflow (for which we shall not assign a different notation) generated by (1.1). An inertial manifold $\mathcal{M}$ for this evolution equation is a subset in $\mathrm{W}$ such that the following conditions are satisfied: $\langle 1\rangle \mathcal{M}$ is a finite dimensional and Lipschitz continuous manifold. $\langle 2\rangle \mathcal{M}$ is positively invariant under the semiflow $S(t)$, i.e. $S(t) \mathcal{M} \subseteq \mathcal{M}$ for $t \geq 0 .\langle 3\rangle \mathcal{M}$ attracts all the trajectories of the equation (1.1) at a uniform exponential rate, i.e. there exists a constant $v>0$ such that for any bounded set $B$ in $H$, there is a constant 
$K(B)$ only depending on $B$ and the distance from $u\left(t, u_{o}\right)$ to the manifold $\boldsymbol{I} L$ decays exponentially ,

$$
\operatorname{dist}\left(\mathrm{u}\left(\mathrm{t}, \mathrm{u}_{\mathrm{o}}\right) ; \mathcal{M}\right) \leq \mathrm{K}(\mathrm{B}) \mathrm{e}^{-v t} \text {, for all } \mathrm{t}>0 \text {, and any } \mathrm{u}_{\mathrm{o}} \in \mathrm{B}
$$

The significance of inertial manifold, if exists, is now well known since it provides a finite dimensional system of ODEs on the inertial manifold, called the inertial form, which lends us to carry on various finite-dimensional analysis on the global dynamics of the original infinite dimensional system (1.1). Recently it has been found, cf. Taboada and You [3], that the inertial manifold theory has an impact on the stabilization of certain nonlinear elastic systems by finite-mode feedback control.

Due to the technical restrictions involved in the currently existing theories of inertial manifolds, typically the spectral gap conditions, the spectral blocking conditions, the large damping condition, or the nonlocal nonlinearity conditions attached to various existence theorems, for many nonlinear higher dimensional parabolic and hyperbolic evolution equations with more or less dissipativity mechanism, the challenging question of whether an inertial manifold exists or not remains unanswered.

As a modest and useful substitute to inertial manifolds, the concept of approximate inertial manifolds (briefly called AIM below) was introduced. In general, an AIM is a subset $\mathcal{M}$ in the state space W, such that

1) $\mathcal{M}$ is a finite dimensional and Lipschitz continuous manifold,

2) $\mathcal{M}$ attracts all the trajectories into a thin neighborhood at a uniform exponential rate,

3) for any trajectory $\left\{u\left(t, u_{0}\right): t \geq 0\right\}$ there is a tracking orbit $\{v(t): t \geq 0\}$ on this manifold, which is governed by a rather simple equation, such that after a transient period $t_{l}(B)$ ( unifirm for $u_{0}$ in a bounded set $B)$ the error $\left\|u\left(t, u_{o}\right)-v(t)\right\|$ is uniformly bounded and decay exponentially to zero.

The existence and construction of AIM are investigated by three major methods: 1$\rangle$ The nonlinear Galerkin approximation method, cf. Temam [2, 4], Foias et al. [5], and Marion [6]. (2) The Gamma method, cf. Sell [7]. 〈3〉 The steady-state determining mapping method which was initiated by Foias et al. $[8,9]$, and developed by Titi [10, 11], and Jolly et al. [12].

It is worth mentioning that in Titi $[10,11]$ the approximate inertial manifolds of the form $\mathcal{M}^{\mathbf{s}}=$ Graph $\left(\Phi^{\mathbf{S}}\right)$ with $\Phi^{\mathbf{S}}$ the staedy-state determining mapping was introduced to 2D Navier-Stokes equations with Dirichlet and periodic boundary conditions. Moreover, in Titi [11] the successive approximation was used to produce a sequence of explicit approximate forms of the mapping $\Phi^{\mathbf{S}}$. These ideas were applied to the 1D Kuramoto-Sivashinsky equation with the periodic boundary conditions by Jolly et al. [12].

This work is to generalize the theory of existence and construction of AIM for the abstract nonlinear parabolic evolution equatiol، (1.1) via the approach of using the steady-state determining mappting, under a unified framework of essentially two fundamental assumptions:

First, in the energy spave $V$ there exists an absorbing set for the concerned semiflow $S(t)$, which is ensured by certain typical hypotheses made in this paper or some alternatives elsewhere. Second, the local (regular) Lipschitz continuity of the nonlinear part R(u): (cf. Assumption (A2))

$$
|R(u)-R(v)| \leq C_{2}(\|u\|,\|v\|)\|u-v\| \text {, for } u \text { and } v \in V \text {, }
$$

which automatically implies that the crucial weak monotonicity (3.10) of the truncated nonlinearity $F(u)$ is satisfied. The main results of this work are stated in Theorem 5.4, of which the essence can be described briefly as: The nonlinear evolution equation (1.1) satisfying the aforementioned two assumptions has AIM in the form of the graph of steady-state determining mapping, and the size of the attracting neighborhood of such AIM shrinks to zero at an explicit fractional power rate.

The work in this paper features the methodology, the decreasing rate estimates of the attracting neighborhood thickness, and the extensive applications of the results (in Theorem 5.4 and Theorem 5.6). 
Specifically, the methodology in proving that the graph of the steady-state determining mapping forms AIM is based on the weak morotonicity (3.10) (which is actually a consequence of the local Lipschitz continuity combined with the dissipativity, rather than a new condition) and on the localizing and pasting technicality in Section 4 and Section 5. This technicality consists of the intervention of an $x$ stationary (or semi-stationary) intermediate equation (4.2), the local estimates $(5.17)$ and $(5.19)$ in breaking terms, and finally the subinterval optimization. Hence the methodology is independent of the concrete or unique properties possessed only by Navier-Stokes equation or by some other equations.

The main results in Theorem 5.4 also include the decreasing rate expressions $(5.15): J(\mu) \approx 1 / \mu^{3 / 2}$ in $\mathrm{H}$ and (5.16): $\mathrm{N}(\mu) \approx 1 / \mu$ in $\mathrm{V}$ of the exponentially attracting neighborhoods of the AIMs. The theory developed in this work is extensively applicable to various dissipative nonlinear parabolic equations, including the 2D Navier-Stokes equations. For different exemplification, in this paper, the applications of this general theory to Burgers' equation, higher dimensional reaction-diffusion equations, 2D GinzburgLandau equations, and axially symmetric Kuramoto-Sivashinsky equations are presented in detail. It is also worth mentioning that the obtained results can be applied to the class of evolution equations studied independently by Smiley [13].

The rest of the paper is organized as follows. In Section 2 the assumptions, the global existence and regularity of solutions, and the existence of absorbing sets are presented. Section 3 deals with the truncation, the decomposition, the steady-state equation, and the existence as well as the Lipschtz continuity of the determining mappings. In Section 4 three technical lemmas involving the semi-stationary intermediate equations are proved. The main theorems on the existence and properties of AIM are provided in Section 5. Finally in Section 6, applications of the obtained results to four different types of nonlinear parabolic equations are illustrated.

\section{SOLUTION SEMIGROUP AND ABSORBING SETS}

Let $\mathbf{H}$ be a real separable Hilbert space and denote its norm and inner-product by $|\cdot|$ and $\langle\cdot$, respectively. We make following assumptions on the linear operator $A$ and the nonlinear part $R(u)$ and $f$ in the abstract evolution equation (1.1).

ASSUMPTION (A1). Assume that $A: D(A)(\subset H) \rightarrow H$ is a closed linear operator, and it is positive definite and self-adjoint, and has compact resolvent..

By this assumption the spectrum set $\sigma(A)$ of $A$ consists of countably many positive eigenvalues $\left\{\lambda_{i}: i=1,2, \ldots\right\}$, each repeated up to its finite multiplicity and $\lim \lambda_{i}=+\infty$ as $i \rightarrow \infty$. The corresponding complete and normalized eigenvectors of $A$ are denoted by $\left\{e_{i}: i=1,2, \ldots\right\}$ which form an orthonormal basis for the space $H$. For any number $s \geq 0$, the fractional power operator $A^{s}: D\left(A^{s}\right) \rightarrow H$ is well defined by the spectral mapping. In particular, we define another real Hilbert space $V$ by $V=D\left(A^{1 / 2}\right)$ with the norm $\|v\|=\left|A^{1 / 2} v\right|$ and the induced inner-product $\langle\langle.,\rangle$.$\rangle . Denote by \lambda_{0}$ the minimum eigenvalue of the operator $A, \lambda_{0}>0$. Note that due to Assumption (A1), - A generates an analytic contraction semigroup denoted by $\{T(t), t \geq 0\}$.

ASSUMPTION (A2). R(.) is a nonlinear mapping from $V$ to $H$ and also from $D(A)$ to $V$, satisfing the following conditions,

$$
\begin{aligned}
& |R(u)| \leq\left(C_{0}(|u|)+k\|u\|\right)\|u\|, \quad \forall u \in V, \\
& \|R(u)\| \leq C_{1}(|u|,\|u\|)|A u|, \forall u \in D(A), \\
& |R(u)-R(v)| \leq C_{2}(\|u\|,\|v\|)\|u-v\|, \forall u, v \in V, \\
& \|R(u)-R(v)\| \leq C_{3}(|A u|,|A v|)|A u-A v|, \forall u, v \in D(A), \\
& \langle R(u), u\rangle \geq \gamma-\beta \mid u 2, \forall u \in V,
\end{aligned}
$$

where $\kappa>0, \gamma \in \mathbf{R}$, and $0<\beta<\lambda_{0}$ are constants, $C_{i}(\ldots), i=0,1,2,3$, are nonnegative continuous functions increasing with their respective variables. Besides assume that $f \in V$ is a time-invariant function. 
REMARK 2.1. The above assumption (A2) on the nonlinearity $R(u)$ is only a typical set of conditions under which one can work out the existence of global solutions over $[0, \infty)$ and the existence of absorbing sets. There are certainly alternative sets of conditions which can replace (A2) in different settings for the same purpose: obtaining the global dissipativity in terms of the absorbing property. After the stage of showing the absorbing property, (A2) plays no more role except the first and third inequality conditions of in (A2).

For any $u_{0} \in H$, the concepts of mild solution, strong solution, and classical solution of the evolution equation (1.1) are provided in Pazy [14]. We refer to Temam [2] for the concept of absorbing set and absorbing property. Following is the main result in this preliminary section.

THEOREM 2.2. For any $u_{o} \in V$, there exists a unique classical solution $u($.$) of the evolution$ equation (1.1) such that

$$
u \in C([0, \infty) ; V) \cap C^{1}((0, \infty) ; H) \cap C((0, \infty) ; D(A))
$$

Moreover, there exists an absorbing set in $\mathrm{V}$ for the semiflow generated by (1.1).

PROOF. We shall use the two basic inequalities, cf. Pazy [14], related to the regularity of an analytic semigroup $\{T(t), t \geq 0\}$ :

$$
\begin{aligned}
& \left\|A^{\alpha} T(t)\right\|_{H \rightarrow H} \leq M_{\alpha} t^{-\alpha} e^{-\omega t}, \text { for all } t>0, \\
& |T(t) x-x| \leq B_{\alpha} t^{\alpha}\left\|A^{\alpha} x\right\|, \text { for all } x \in D\left(A^{\alpha}\right) \text { and } t \geq 0,
\end{aligned}
$$

where $0 \leq \alpha \leq 1, M_{\alpha}$ and $B_{\alpha}$ are constants depending on $\alpha$, and $\omega$ satisfies $0<\omega<\lambda_{0}$. Below we sketch the proof of this theorem by several steps.

STEP 1 . The local existence and uniqueness of a mild solution on some interval $[0, \tau]$. This can be done by considering the integral equation

$$
v(t)=A^{1 / 2} T(t) u_{o}+\int_{0}^{t} A^{1 / 2} T(t-s)\left[f-R\left(A^{-1 / 2} v(s)\right)\right] d s, \text { for } t \in[0, \tau],
$$

and using the contraction mapping and fixed point argument to choose small $\tau>0$ such that there exists a unique strongly continuous solution $v\left(\right.$.) oi the equation (2.4), so that $u(t)=A^{-1 / 2} v(t), t \in[0, \tau]$, is the mild solution of $(1.1)$ and $u \in C([0, \tau] ; V)$.

STEP 2. The regularity of the solution $u($.$) . We want to show that u(t) \in D(A)$ for $t \in(0, \tau]$. By the mild solution formula

$$
u(t)=T(t) u_{o}+\int_{0}^{t} T(t-s)[f-R(u(s))] d s, \quad \text { for } t \in[0, \tau],
$$

and the property (2.2), we need only to consider the nonlinear term

$$
g(t)=\int_{0}^{t} T(t-s) R(u(s)) d s=\int_{0}^{t} T(t-s)[R(u(s))-R(u(t))] d s+\int_{0}^{t} T(t-s) R(u(t)) d s,
$$

in which

$$
A \int_{0}^{t} T(t-s) R(u(t)) d s=[T(t)-I] R(u(t))
$$




$$
\int_{0}^{t} T(t-s)[R(u(s))-R(u(t))] d s=\lim _{\varepsilon \rightarrow 0} h_{\varepsilon}(t)
$$

where $0<\varepsilon<\mathrm{t}$,

and

$$
h_{\varepsilon}(t)=\int_{0}^{t-\varepsilon} T(t-s)[R(u(s))-R(u(t))] d s \in D(A),
$$

$$
\begin{aligned}
\lim _{\varepsilon \rightarrow 0} A h_{\varepsilon}(t) & =\lim _{\varepsilon \rightarrow 0} \int_{0}^{t} x[0 . t-\varepsilon](s) A T(t-s)[R(u(s))-R(u(t))] d s \\
& =\int_{0}^{t} \operatorname{AT}(t-s)[R(u(s))-R(u(t))] d s
\end{aligned}
$$

where the convergence in (2.8) follows from the dominate convergence theorem and

$$
|X[0 . t-\varepsilon)(s) A T(t-s)[R(u(s))-R(u(t))]| \leq \text { const }|t-s|^{\delta-1}|s|^{\delta} \in L(0, t),
$$

which in turn comes from (2.2) and the local Holder continuity of the solution $u$, (the proof of the latter is based upon the basic semigroup properties (2.2) and (2.3), and omitted here), namely, there exist a constant $0<\delta<1 / 2$, and a constant $C\left(\tau, u_{0}, f, \delta\right)>0$, such that

$$
\|u(s)-u(t)\| \leq C\left(\tau, u_{0}, f, \delta\right)|s-t| \delta|s|-\delta, \quad \text { for } 0<s \leq t \leq \tau .
$$

Thanks to the closedness of $A,(2.8)$ implies that

$$
\int_{0}^{t} T(t-s)[R(u(s))-R(u(t))] d s \in D(A) .
$$

As a consequence, (2.7) and (2.9) im, lies that $g(t) \in D(A)$ and that $\mathrm{Ag}(\mathrm{t})$ is continuous on $[0, \tau]$. Furthermore,by Theorem 4.2.4 of Pazy [14], this implies that $u \in \mathrm{Cl}^{l}((0, \tau] ; \mathrm{H})$.

STEP 3. The global Existence and absorbing property of solutions in $\mathrm{H}$. We shall make a priori estimates to show these two things together. First, assume that $u_{0} \in D(A)$. Then the corresponding local solution exists in $C([0, \tau\} ; D(A)) \cap C^{1}([0, \tau] ; H)$ and its right $H$-strong derivative at $t=0$ satisfies the differential equation (1.1) for $t=0$. Take the inner-product of the equation (1.1) with the solution itself, we get

$$
\frac{1}{2} \frac{d}{d t}|u(t)|^{2}+\|u(t)\|^{2}-\beta|u(t)|^{2}+\gamma \leq\langle f, u(t)\rangle \leq \varepsilon|u(t)|^{2}+\varepsilon^{-1}|f|^{2}
$$

for all $t \in\left[0, T_{m}\right)$, the maximal interval of existence, with $T_{m}=T_{m}\left(u_{o}\right)$, where we let $0<\varepsilon<\frac{1}{2}\left(\lambda_{o}-\beta\right)$ and fix it, so that

$$
\frac{d}{d t}|u(t)|^{2}+\left(\lambda_{0}-\beta\right)|u(t)|^{2} \leq 2|\gamma|+2 \varepsilon^{-1}|f|^{2}
$$

Integrating this differential inequality, we obtain

$$
|u(t)|^{2} \leq \exp \left(-\left(\lambda_{0}-\beta\right) t\right)\left|u_{o}\right|^{2}+K_{0}(f), \text { for } t \in\left[0, T_{m}\right),
$$


where

$$
K_{o}(f)=\left(\lambda_{0}-\beta\right)^{-1}\left(2 \gamma+2 \varepsilon^{-1}|f|^{2}\right)
$$

is a constant independent of $\mathrm{u}_{0}$. Then by the denseness of $\mathrm{D}(\mathrm{A})$ in $\mathrm{V}$ and the approximation procedure with the aid of continuous dependence property of solutions on the initial data, we can assert that (2.12) holds for all the solutions with $u_{0} \in V$. Hence, the solution claimed in STEP 1 exists uniquely and globally on $[0, \infty)$. Moreover (2.12) shows that

$$
\lim \operatorname{up}_{t \rightarrow \infty}|u(t)|^{2} \leq K_{o}(f),
$$

which means that there is an absorbing set, e.g. the closed ball in $\mathrm{H}$ centered at the origin and of radius $\mathrm{K}_{\mathrm{o}}(\mathrm{f})+1$.

STEP 4. The global existence and absorbing property of solutions in $\mathrm{V}$. We shall use the same procedure that first assume that $u_{0}$ in $D(A)$ and at last extend the integrated result to all $u_{0}$ in $V$ through approximation. Thus, for $u_{0} \in D(A)$, take the inner-product of the equation (1.1) with $A u(t)$ and use the first two inequality conditions of Assumption (A2) to obtain

$$
\begin{aligned}
& \quad \frac{1}{2} \frac{d}{d t}\|u(t)\|^{2}+|A u(t)|^{2} \leq|R(u(t))|^{2}+\frac{1}{2}|f|^{2}+\frac{1}{2}|A u(t)|^{2} \\
& \leq 2\left\{C_{0}(|u(t)|)^{2}+k^{2}\|u(t)\|^{2}\right\}\|u(t)\|^{2}+\frac{1}{2}|f|^{2}+\frac{1}{2}|A u(t)|^{2},
\end{aligned}
$$

so that

$$
\frac{d}{d t}\|u(t)\|^{2} \leq \varphi(t)\|u(t)\|^{2}+|f|^{2}, \text { for } t \in\left[0, T_{m}\right)
$$

with

$$
\varphi(t)=4 C_{0}\left(\exp \left(-\frac{1}{2}\left(\lambda_{0}-\beta\right) t\right)\left|u_{0}\right|+\sqrt{K_{0}(f)}\right)^{2}+4 k^{2}\|u(t)\|^{2}
$$

From (2.11) through (2.13), we have

$$
\begin{aligned}
& \int_{t}^{t+r} \| u(s)||^{2} d s \leq \frac{1}{2}|u(t)|^{2}+(\beta+1) \int_{t}^{t+r}|u(s)|^{2} d s+\left(|\gamma|+|f|^{2}\right) r \\
& \quad \leq \exp \left(-\left(\lambda_{0}-\beta\right) t\right)\left[\frac{1}{2}+(\beta+1)\left(\lambda_{0}-\beta\right)^{-1}\right]\left|u_{0}\right|^{2}+K_{1}(f)
\end{aligned}
$$

for $[t, t+r]$ in $\left[0, T_{m}\right)$, where

$$
K_{1}(f)=(1 / 2) K_{0}(f)+\left[(\beta+1) K_{0}(f)+|\gamma|+|f|^{2}\right] r .
$$

From (2.17) it follows that

$$
\int_{t}^{t+r} \varphi(s) d s \leq d\left(r, u_{0}, f\right)(\text { constant }), \text { for } 0 \leq t \leq t+r<T_{m} .
$$

Apply the uniform Gronwall inequality to (2.16), we get

$$
\begin{gathered}
\|u(t+r)\|^{2} \leq \exp \left(d\left(r, u_{0}, f\right)\right)\left\{r|f|^{2}+r^{-1} K_{1}(f)\right. \\
\left.+r^{-1}\left[\frac{1}{2}+(\beta+1)\left(\lambda_{0}-\beta\right)^{-1}\right]\left|u_{o}\right|^{2} \exp \left(-\left(\lambda_{0}-\beta\right) t\right)\right\}
\end{gathered}
$$


for $0 \leq t \leq t+r<T_{m}$. Since $r>0$ can be fixed, say, $r \in\left(0, \frac{1}{3} T_{m}\right)$. The above inequality (2.21) shows that the solution $u(t)$ remains bounded in $V$ as $t+r \rightarrow T_{m}$. Therefore the solution claimed in STEP 1 exists uniquely and globally in $\mathrm{V}$, in other words, $\mathrm{T}_{\mathrm{m}}=+\infty$. Moreover, note that

$$
\lim _{t \rightarrow \infty} \int_{t}^{t+r} \varphi(s) d s=K_{2}(f), \text { with } K_{2}(f)=4 r C_{o}\left(\sqrt{K_{o}(f)}\right)^{2}+4 k^{2} r K_{1}(f),
$$

where the convergence is uniform for $\left|u_{0}\right|$ in any given bounded set. From (2.20) through (2.22) we have

$$
\lim \sup _{t \rightarrow \infty}\|u(t)\|^{2} \leq K_{3}(f), \text { with } K_{3}(f)=\left[r|f|^{2}+r^{-1} K_{1}(f)\right] \exp \left(K_{2}(f)\right)
$$

It means that there is an absorbing set in $\mathrm{V}$ for this semiflow, and the absorbing set can be a ball centered at the origin and of radius $\rho=K_{3}(f)^{1 / 2}+\eta$, for any positive constant $\eta$. The proof of Theorem 2.1 is completed.The proof is copmpleted.

REMARK 2.3. Concerning the monotone condition of Assumption (A2):

$$
\langle\mathbf{R}(\mathbf{u}), \mathbf{u}\rangle \geq \gamma-\beta|\mathbf{u}|^{2}, \text { for all } \mathbf{u} \in \mathrm{V},
$$

with $0<\beta<\lambda_{0}$, we note that if $R($.$) satisfies the following condition,$

$$
\langle R(u)-R(v), u-v\rangle \geq 0, \forall u, v \in V \text { and }\|u-v\|>d_{o}
$$

where $d_{0}>0$ is a constant, then the alove monotone condition is automatically satisfied. In fact, let $m_{0}=$ $\sup \left\{|R(u)|:\|u\| \leq d_{0}\right\}<\infty$. We have: i) for any $u \in V$ such that $\|u\| \leq d_{0}$,

$$
\langle R(u), u\rangle \geq-|R(u)||u| \geq-m_{o} d_{o} \lambda_{0}{ }^{1 / 2}
$$

ii) for any $u \in V$ such that $\|u\|>d_{0}$,

$$
\langle\mathbf{R}(\mathrm{u}), \mathrm{u}\rangle=\langle\mathbf{R}(\mathrm{u})-\mathrm{R}(0), \mathrm{u}-0\rangle+\langle\mathrm{R}(0), \mathrm{u}\rangle \geq-|\mathrm{R}(0)||\mathrm{u}| \geq-\frac{1}{\varepsilon}|\mathrm{R}(0)|^{2}-\varepsilon|\mathrm{u}|^{2},
$$

in which one can take $\varepsilon$ sstisfying $0<\varepsilon<\lambda_{0}$. Therefore the monotone condition of (A2) is satisfied with the parameters $\beta=\varepsilon$ and $\gamma=\max \left\{\varepsilon^{-1}|R(0)|^{2}, \bmod _{0} \lambda_{0} 1 / 2\right\}$.

\section{STEADY-STATE DETERMINING MAPPINGS}

In this section we make some preprations to construct approximate inertial manifolds: truncation, space decomposition, and define the steady-state determining mappings.

TRUNCATION. Fix a constant $\rho>0$ such that $\rho^{2}>K_{3}(f)$, with $K_{3}(f)$ defined by (2.23). Define a $C^{1}$ scalar function $\theta(r):[0, \infty) \rightarrow[0,1]$, which satisfies the following conditions,

$$
\begin{gathered}
\theta(r)=1, \text { for } 0 \leq r \leq 1, \\
\theta(r) \equiv 0, \text { for } r \geq 3, \\
0 \leq \theta(r) \leq 1 \text {, and }\left|\theta^{\prime}(r)\right| \leq 1 \text {, elsewhere. }
\end{gathered}
$$

Let $\theta_{\rho}(r)=\theta\left(\frac{r}{\rho^{2}}\right)$ with $\rho$ being the fixed constant mentioned above. Define a new nonlinear mapping by

$$
F(u)=\theta_{\rho}\left(\|u\|^{2}\right) R(u), \text { for } u \in V \text {. }
$$


In the sequal we shall consider the modified equation:

$$
\underset{d t}{d u}+A u+F(u)=f, t \geq 0, u(0)=u_{o} \in V
$$

By the result of STEP 4 in the proof of Theorem 2.1, specifically (2.23), the ball $\mathrm{B}(0 ; \rho)$ centered at the origin and of radius $\rho$ is an absorbing set for the semiflow in $\mathrm{V}$ generated by the equation (1.1). Therefore this truncation that replacing $(1.1)$ by $(-2)$ preserves the long-time dynamics of the original equation (1.1), since within the absorbing ball $\mathrm{B}(0 ; \rho)$ both equations are the same. But the new truncated nonlinearity has following uniform properties in which $c_{1}>0$ and $c_{2}>0$ are two uniform constants:

$$
\begin{aligned}
& |F(u)| \leq c_{1}\|u\|, \quad \text { for any } u \in V, \\
& \|F(u)\| \leq c_{1}|A u|, \text { for any } u \in D(A), \\
& |F(u)-F(v)| \leq c_{2}\|u-v\|, \quad \text { for any } u \text { and } v \text { in } V, \\
& \|F(u)-F(v)\| \leq c_{2}|A u-A v|, \text { for any } u \text { and } v \text { in } D(A) \text {. }
\end{aligned}
$$

DECOMPOSITION. For any given positive number $\mu>\lambda_{0}$, there exist a nontrivial spectral decomposition of $\sigma(\mathrm{A})$ and a corresponding orthogonal decomposition of the Hilbert spaces $\mathrm{H}$ and $\mathrm{V}$ :

$$
\sigma(A)=\sigma_{\mu}^{+}(A) \cup \sigma_{\mu}^{-}(A),
$$

$H=X_{\mu}+Y_{\mu}$, (denoted briefly by $X+Y$ if $\mu$ is relatively fixed),

$V=X_{\mu}+Z_{\mu}$, (denoted briefly by $X+Z$ if $\mu$ is relatively fixed),

where

$$
\sigma_{\mu}^{+}(\mathbf{A})=\{\lambda \in \sigma(\mathbf{A}): \lambda \geq \mu\}, \sigma_{\mu}(\mathbf{A})=\{\lambda \in \sigma(\mathbf{A}): \lambda<\mu\},
$$

$X_{\mu}=\operatorname{Span}\left\{e_{i}: 1 \leq i \leq n(\mu)\right\}, Y_{\mu}=C_{H} \operatorname{Span}\left\{e_{i}: i>n(\mu)\right\}$, and $Z_{\mu}=C l_{V} \operatorname{Span}\left\{e_{i}: i>n(\mu)\right\}$, in which $n(\mu)=\max \left\{i\right.$ (integer): $\left.\lambda_{i}<\mu\right\}$. Let $P: H \rightarrow X$ be the orthogonal projection and $Q_{H}=I_{H}-P$. Then $X=$ $\mathrm{PH}, \mathrm{Y}=\mathrm{QH}$, and $\mathrm{Z}=\mathrm{QV}$. Apply the projections $\mathrm{P}$ and $\mathrm{Q}$ to the equation (3.2), we get two coupled equations:

$$
\begin{aligned}
& \frac{d x}{d t}+A x+P F(x+z)=g=P f, \quad x(0)=x_{0}=P u_{o} \in X \\
& \frac{d z}{d t}+A z+Q F(x+z)=h=Q f, \quad z(0)=z_{0}=Q u_{0} \in Z
\end{aligned}
$$

DETERMINING MAPPTINGS. Consider the associated algebraic equation

$$
A \mathbf{u}+F(u)=\mathbf{f}
$$

which is called the steady state equation. It can also be decomposed as

$$
\begin{aligned}
& A x+P F(x+z)=g \\
& A z+Q F(x+z)=h
\end{aligned}
$$

with $x \in X_{\mu}$ and $z \in Z_{\mu}$. Suppose that for any given $h \in Z_{\mu}$ and any $x \in X_{\mu}$, the component equation (3.7)z has a unique solution $z$, denoted by

$$
z=\varphi(x, h),
$$


then the solution of the steady state equation (3.6) reduces to the solution of the following equation,

$$
A x+P F(x+\varphi(x, h))=g \text {, in } X_{\mu},
$$

which is called, according to Chow and Hale [15], a bifurcation equation. The mapping $\varphi: X_{\mu} \times Z_{\mu} \rightarrow Z_{\mu}$ defined by (3.8), if exists, is called a steady-state determining mapping.

In order to study the existence and properties of such determining mappings that will be a tool in contructing AIMs, we now emphasize an important consequent property derived from the uniform Lipschiotz continuity of (3.3) as follows.

LEMMA 3.1. Under Assumption (A2) and the truncation (3.1), the truncated nonlinearity F(u) satisfies the following weak monotonicity :

$$
\langle F(u)-F(v), u-v\rangle \geq-c_{2}\|u-v\||u-v|, \quad \text { for any } u, v \in V,
$$

where $c_{2}>0$ is the Lipschitz constant in (3.3).

PROOF. By (3.3) the truncated nonlinearity $F(u)$ possesses the uniform Lipschitz continuity $\mid F(u)$ - $F(v) \mid \leq c_{2}\|u-v\|$, for any $u$ and $v$ in $V$. Thus we have

$$
\langle F(u)-F(v), u-v\rangle \geq-|F(u)-F(v)||u-v| \geq-c_{2}\|u-v\||u-v|, \text { for } u, v \in V \text {. }
$$

This indicates that (3.10) holds.

THEOREM 3.2. For any $\mu>\left(c_{2}\right)^{2}$ and the decomposition (3.4) and (3.7) associated with this $\mu$, there exists a Lipschitz continuous mapping $\varphi: X \times Z \rightarrow Z$, such that

$$
\mathbf{z}=\varphi(\mathbf{x}, \mathbf{h})
$$

is a unique solution of the equation (3.7)Z. Moreover, this mapping $\varphi$ has the following properties,

$$
\begin{aligned}
& \left|\varphi\left(\mathbf{x}_{1}, \mathrm{~h}_{1}\right)-\varphi\left(\mathbf{x}_{2}, \mathrm{~h}_{2}\right)\right| \leq \frac{1}{\mu-\mathbf{c}_{2} \mu^{1 / 2}}\left[\mathbf{c}_{2}\left\|\mathbf{x}_{1}-\mathbf{x}_{2}\right\|+\mu^{-1 / 2}\left\|\mathbf{h}_{1}-\mathbf{h}_{2}\right\|\right], \\
& \left\|\varphi\left(\mathbf{x}_{1}, h_{1}\right)-\varphi\left(\mathbf{x}_{2}, h_{2}\right)\right\| \leq \frac{1}{\mu^{1 / 2}-c_{2}}\left[c_{2}\left\|\mathbf{x}_{1}-\mathbf{x}_{2}\right\|+\mu^{-1 / 2}\left\|h_{1}-h_{2}\right\|\right] .
\end{aligned}
$$

PROOF. Define a mapping G: $D(A) \cap Q V \rightarrow Q V$ by

$$
G: \mathbf{w} \rightarrow A_{Z} \mathbf{w}+Q F(x+w) .
$$

where $A_{Z}=A \mid Z$ (the restriction of $A$ on $Z$ ). $G$ is a densely defined operator in $Q V$. We want to show that $G$ is surjective, i.e. $\operatorname{Ran}(G)=Q V$. For any $h \in Q V$, the equation $G w=h$ is solvable if and only if the following equation is solvable:

$$
w=\left(A_{Z}\right)^{-1}[h-Q F(x+w)] \text { for } w \in Q V .
$$

Define a mapping $\mathrm{J}: \mathrm{QV} \rightarrow \mathrm{QV}$ by

$$
J(w)=\left(A_{Z}\right)^{-1}[h-Q F(x+w)] .
$$

Since we have

$$
\begin{gathered}
\left\|J\left(w_{1}\right)-J\left(w_{2}\right)\right\| \leq\left\|\left(A_{Z}\right)^{-1}\right\|_{L(H ; V)}\left|Q F\left(x+w_{1}\right)-Q F\left(x+w_{2}\right)\right| \\
\quad \leq\left\|\left(A_{Z}\right)^{-1}\right\|_{L(H ; V)} c_{2}\left\|w_{1}-w_{2}\right\| \leq \frac{c_{2}}{\mu^{1 / 2}}\left\|w_{1}-w_{2}\right\|
\end{gathered}
$$


where $\left\|\left(A_{Z}\right)^{-1}\right\|_{L(H ; V)} \leq \mu^{-1 / 2}$ can be directly verified by the eigen-expansion. Since $\mu^{1 / 2}>c_{2},(3.17)$ shows that $J$ is a contraction mapping so that it has a unique fixed point $w$ for any given pair $(x, h) \in X \times$ $Z$. Therefore (3.15) is solvable and the equation (3.7) $Z$ has a unique solution $z$, denoted by (3.11).

Next we prove the Lipschitz continuity of this mapping $\varphi:(x, h) \rightarrow z$. Let $z_{i}=\varphi\left(x_{i}, h_{i}\right), i=1,2$. It holds that

$$
\begin{gathered}
\left(\mu-c_{2} \mu^{1 / 2}\right)\left|z_{1}-z_{2}\right|^{2} \leq\left(\mu^{1 / 2}-c_{2}\right)|| z_{1}-z_{2} \|\left|z_{1}-z_{2}\right| \\
\leq\left\|z_{1}-z_{2}\right\|^{2}+\left\langle Q F\left(x_{1}+z_{1}\right)-Q F\left(x_{1}+z_{2}\right), z_{1}-z_{2}\right\rangle \\
s\left\langle A\left(z_{1}-z_{2}\right), z_{1}-z_{2}\right\rangle+\left\langle Q F\left(x_{1}+z_{1}\right)-Q F\left(x_{2}+z_{2}\right), z_{1}-z_{2}\right\rangle \\
+\left\langle Q F\left(x_{2}+z_{2}\right)-Q F\left(x_{1}+z_{2}\right), z_{1}-z_{2}\right\rangle \\
\leq\left\langle h_{1}-h_{2}, z_{1}-z_{2}\right\rangle+c_{2}\left\|x_{1}-x_{2}\right\|\left|z_{1}-z_{2}\right|
\end{gathered}
$$

which implies (3.12) and (3.13):

$$
\begin{gathered}
\left|z_{1}-z_{2}\right| \leq \frac{1}{\mu-c_{2} \mu^{1 / 2}}\left[c_{2}\left\|x_{1}-x_{2}\right\|+\mu^{-1 / 2}\left\|h_{1}-h_{2}\right\|\right] . \\
\left\|z_{1}-z_{2}\right\| \leq \frac{1}{\mu^{1 / 2}-c_{2}}\left[c_{2}\left\|x_{1}-x_{2}\right\|+\mu^{-1 / 2}\left\|h_{1}-h_{2}\right\|\right] .
\end{gathered}
$$

The proof is completed.

We call the mapping $\varphi$ obtained in this theorem as the determining mapping associated with $\mu$.

\section{TECHNICAL LEMMAS}

In this section three lemmas will be established as intermediate steps toward the main result. First we sketch the guiding procedure leadin r to the construction of AIMs as follows. In fact we look for an AIM in the form of

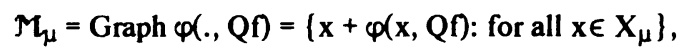

where $\mu>0$ is to be chosen. Since an AIM needs not to be invariant, for the finite dimensional and Lipschitz continuous manifold $\mathcal{M}_{\mu}$ defined by $(4.1)$, the only thing to be done is the exponential attraction of any orbits to a "thin" neighborhood of this manifold $\mathcal{M}_{\mu}$. In order to prove this attraction property, naturally we shall try to estimate the distance between the Z-component $z(t)$ of the original solution $u(t)$ and the image $\varphi(x(t), Q f)$ of the X-component $x(t)$ of $u(t)$, since $x(t)+\varphi(x(t), Q f)$ is on the manifold $M_{\mu}$. Such an estimate is based on the following lemmas.

LEMMA 4.1. Assume that $\mu>\left(c_{2}\right)^{2}$. Let $x \in X_{\mu}$ and $h \in Z_{\mu}$. If $z(.) \in C\left([0, \tau) ; Z_{\mu}\right)$ for some $\tau>$ 0 is a classical solution of the following evolution equation

$$
\frac{d z}{d t}+A z+Q F(x+z(t))=h, \text { for } t \in[0, \tau)
$$

then the following estimates are valid,

$$
\begin{aligned}
& |z(t)-\varphi(x, h)| \leq|z(0)-\varphi(x, h)| \exp \left(-\left(\mu-c_{2} \mu^{1 / 2}\right) t\right), \text { for } t \in[0, \tau), \\
& \|z(t)-\varphi(x, h)\| \leq\|z(0)-\varphi(x, h)\| \exp \left(-\frac{1}{2}\left(\mu-\left|c_{2}\right|^{2}\right) t\right), \text { for } t \in[0, \tau),
\end{aligned}
$$

in which $\varphi$ is the determining mapping associated with the given $\mu$. 
PROOF. Define a functional $I I(z)=|z-\varphi(x, h)|^{2}$, for $z \in Z_{\mu}$. For the classical solution $z(t)$ of the equation (4.2), we have

$$
\begin{gathered}
\underset{d t}{d} I I(z(t))=2\langle z(t)-\varphi(x, h), h-A z(t)-Q F(x+z(t))\rangle \\
=-2\langle z(t)-\varphi(x, h), A(z(t)-\varphi(x, h))+Q F(x+z(t))-Q F(x+\varphi(x, h))\rangle \\
\leq-2\left(\mu^{1 / 2} \quad c_{2}\right)\|z(t)-\varphi(x, h)\||z(t)-\varphi(x, h)| \\
\leq-2\left(\mu-c_{2} \mu^{1 / 2}\right) I I(z(t)), \text { for } t \in[0, \tau) .
\end{gathered}
$$

Since the classical solution $z(t)$ is absolutely continuous on any interval $\left[t_{0}, t_{1}\right] \subset[0, \tau)$, it follows that

$$
I I(z(t)) \leq I I\left(z\left(t_{0}\right)\right) \exp \left(-2\left(\mu-c_{2} \mu^{1 / 2}\right) t\right), \text { for } t \in[0, \tau)
$$

This leads directly to the estimate (4.3).

Similarly, consider another functional $L(z)=\|z-\varphi(x, h)\|^{2}$ for $z \in Z_{\mu}$. Then we have

$$
\begin{gathered}
\frac{d}{d t} L(z(t))=2\langle\langle z(t)-\varphi(x, h), h-A z(t)-Q F(x+z(t))\rangle\rangle \\
=-2\langle\langle z(t)-\varphi(x, h), A(z(t)-\varphi(x, h))+Q F(x+z(t))-Q F(x+\varphi(x, h))\rangle\rangle \\
=-2|A(z(t)-\varphi(x, h))|^{2}-2\langle A(z(t)-\varphi(x, h)), Q F(x+z(t))-Q F(x+\varphi(x, h))\rangle \\
s-|A(z(t)-\varphi(A, h))|^{2}+|F(x+z(t))-F(x+\varphi(x, h))|^{2} \\
\leq-\left(\mu-\left|c_{2}\right|^{2}\right)\|z(t)-\varphi(x, h)\|^{2}=-\left(\mu-\left|c_{2}\right|^{2}\right) L(z(t)), \text { for } t \in[0, \tau) .
\end{gathered}
$$

After integration of (4.7), we obtain the estimate (4.4).The proof is completed.

COROLLARY 4.2. If $\lambda_{0}>\left(c_{2}\right)^{2}$, then by taking $\mu=\lambda_{0}$ we have $V=Z$ and $X=\{0\}$, and all the solutions $u($.$) of the equation (3.2) satisfy$

$$
\|u(t)-\varphi(0, f)\|^{2} \leq\left\|u_{0}-\varphi(0, f)\right\|^{2} \exp \left(-\frac{1}{2}\left(\mu-\left|c_{2}\right|^{2}\right) t\right), \text { for } t \geq 0 .
$$

Therefore the steady state $\varphi(0, f)$ is globally and exponentially stable in $V$.

Next, let $u(t)=x(t)+z(t), t \geq 0$, with $u(0)=u_{0}$, be any solution of the equation (3.2). Here $x(t)$ $=\mathrm{Pu}(\mathrm{t})$ and $z(t)=\mathrm{Qu}(\mathrm{t})$ with $\mathrm{P}: \mathrm{H} \rightarrow \mathrm{X}=\mathrm{X}_{\mu}$ the orthogonal projection associated with the decompositions (3.4) for some given $\mu>0$.

Let $t^{0}>0$ be arbitrarily given. Denote by $x^{0}=x\left(t^{\circ}\right)$ and $z^{0}=z\left(t^{0}\right)$. We shall estimate the difference between the solutions of following two equations with the same initial data:

$$
\begin{aligned}
& \frac{d z}{d t}+A z+O F(x(t)+z)=h, \quad z\left(t^{0}\right)=z^{0}, t \geq t^{\circ}, \\
& \frac{d w}{d t}+A w+Q F\left(x^{0}+w\right)=h, w\left(t^{\circ}\right)=z^{0}, t \geq t^{\circ},
\end{aligned}
$$

where $A=A_{Z}$. Note that (4.9) is exactly the second component equation (3.7) $Z$ with an initial value condition at $t^{\circ}$. Also note that the truncated nonlinear mapping $F$ has the uniform bound and the uniform 
Lipschitz continuity, so that (referring to the proof of Theorem 2.1) the classical solution $w($.$) of the$ equation (4.10) exists uniquely for $t \in\left[t^{\circ}, \infty\right)$ and has the same regularity as the solution $z($.$) of the$ equation (4.9) has.

We need an auxiliary lemma as follows.

LEMMA 4.3. After any solution $u(t)$ of the original equation $(1.1)$ with $u(0)=u_{0}$ enters the absorbing ball $B(0 ; \rho) \subset V$ for ever, the solution is uniformely Holder continuous with the exponent $1 / 2$, i.e. there is a constant $q>0$, such that for any solution $u(t)$ of $(1.1)$,

$$
\|u(t)-u(s)\| \leq q|t-s|^{1 / 2}, \text { for } t, s \in\left[t_{0}\left(u_{o}\right), \infty\right),
$$

where $t_{0}\left(u_{0}\right)$ is the final entering time of the trajestory $u(t)$ started from $u_{o}$ into the absorbing ball.

PROOF. Assume that $t \geq s \geq t_{0}=t_{0}\left(u_{0}\right)$. We $t_{\text {. }}$ 've

$$
\begin{gathered}
\|u(t)-u(s)\| \leq\|[T(t-s)-I] u(s)\|+\int_{s}^{t}\left\|A^{1 / 2} T(t-\sigma)\right\|_{L(H)}|f| d \sigma \\
\quad+\int_{s}^{t}\left\|A^{1 / 2} T(t-\sigma)\right\|_{L(H)}|F(u(\sigma))| d \sigma \\
\quad \leq B_{1 / 2} \rho|t-s|^{1 / 2}+2 M_{1 / 2}|t-s|^{1 / 2}\left(|f|+\rho c_{1}\right),
\end{gathered}
$$

where (2.2) and (2.3) are used. Thus (4.11) is valid with $q=\rho\left(B_{1 / 2}+2 M_{1 / 2} c_{1}\right)+2 M_{1 / 2}|f|$. The proof is completed.

LEMMA 4.4. Let $z($.$) and w($.$) be the solution of (4.9) and of (4.10) respectively. If \mu>\left(c_{2}\right)^{2}$, then for any given $\Delta>0$,

$$
|z(t)-w(t)| \leq \frac{q c_{2}}{\mu-c_{2} \mu^{1 / 2}} \Delta^{1 / 2}, \quad \text { for } t \in\left[t^{\circ}, t^{o}+\Delta\right], \text { with } t^{o} \geq t_{0}\left(u_{0}\right) .
$$

If $\mu>2\left(c_{2}\right)^{2}$, then for any given $\Delta>0$,

$$
\|z(t)-w(t)\| \leq \sqrt{\frac{2}{\mu-2\left(c_{2}\right)^{2}}} q c_{2} \Delta^{1 / 2}, \text { for } t \in\left[\mathfrak{t}^{\circ}, t^{0}+\Delta\right] \text {, with } t^{0} \geq t_{0}\left(u_{o}\right) \text {. }
$$

Here $q$ and $t_{0}\left(u_{0}\right)$ are the same as described in Lemma 4.3.

Proof. Let $v(t)=z(t)-w(t)$. For $t \in\left[t^{0}, t^{0}+\Delta\right]$, with $t^{0} \geq t_{0}\left(u_{o}\right)$,

$$
\begin{aligned}
& \frac{d}{d t}|v(t)|^{2}=2\left\langle v(t),-A v(t)-Q F(x(t)+z(t))+Q F\left(x^{0}+w(t)\right)\right\rangle \\
& =-2\{\langle A v(t), v(t)\rangle+\langle F(x(t)+z(t))-F(x(t)+w(t)), v(t)\rangle\} \\
& -2\left\langle F(x(t)+w(t))-F\left(x^{0}+w(t)\right), v(t)\right\rangle \\
& \leq-2\left(\mu^{1 / 2}-c_{2}\right)\|v(t)\||v(t)|+\varepsilon|V(t)|^{2}+\varepsilon^{-1}\left|F(x(t)+w(t))-F\left(x^{o}+w(t)\right)\right|^{2} \\
& s-2\left(\mu-c_{2} \mu^{1 / 2}-\varepsilon / 2\right)|v(t)|^{2}-\varepsilon^{-1}\left(c_{2}\right)^{2}\left\|x(t)-x^{\circ}\right\|^{2} \\
& \leq-2\left(\mu-c_{2} \mu^{1 / 2}-\varepsilon / 2\right)|v(t)|^{2}+\varepsilon^{-1}\left(c_{2}\right)^{2} q^{2} \Delta,
\end{aligned}
$$


where we used (4.11) in the last inequality. Take $\varepsilon=\mu-c_{2} \mu^{1 / 2}$, from (4.15) we obtain

$$
\underset{d t}{d}|v(t)|^{2} \leq-\left(\mu-c_{2} \mu^{1 / 2}\right)|v(t)|^{2}+\underset{\mu-c_{2} \mu^{1 / 2}}{\left(c_{2}\right)^{2} q^{2}} \text {, for } t \in\left[t^{\circ}, t^{o}+\Delta\right] .
$$

Integrating this differential inequality, we get

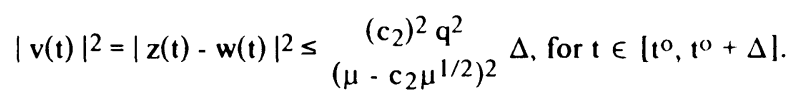

Therefore (4.13) is valid. Moreover, if $\mu>2\left(c_{2}\right)^{2}$, then we have

$$
\begin{aligned}
& \frac{d}{d t}\|v(t)\|^{2}=2\left\langle\left\langle v(t),-A v(t)-Q F(x(t)+z(t))+Q F\left(x^{o}+w(t)\right)\right\rangle\right\rangle \\
&=-|\operatorname{Av}(t)|^{2}+\left|F(x(t)+z(t))-F\left(x^{o}+w(t)\right)\right|^{2} \\
& \leq-\mu\|v(t)\|^{2}+\left(c_{2}\right)^{2}\left[\left\|x(t)-x^{o}\right\|+\|v(t)\|\right]^{2} \\
& S-\left(\mu-2\left(c_{2}\right)^{2}\right)\|v(t)\|^{2}+2\left(c_{2}\right)^{2}\left\|x(t)-x^{o}\right\|^{2} \\
& S-\left(\mu-2\left(c_{2}\right)^{2}\right)\|v(t)\|^{2}+2\left(c_{2}\right)^{2} q^{2} \Delta, \text { for } t \in\left[\mathfrak{r}^{\circ}, t^{0}+\Delta\right] .
\end{aligned}
$$

Integrating this differential inequality, we obtain (4.14). The proof is completed.

\section{APPROXIMATE INERTIAL MANIFOLDS}

In this section we shall prove the existence of approximate inertial manifolds for the original equation (1.1) by the approach of cons'ruction based on the determining mapping $\varphi$. First of all, we define an approximate inertial manifold as follows.

DEFINITION 5.1. A subset $\mathcal{M} \subset \mathrm{V}$ is called an approximate inertial manifold (AIM) for the semiflow generated by the evolution equation (1.1) in $\mathrm{V}$, if $\mathcal{M}$ satisfies the following conditions :

a) $\mathcal{M}$ is a finite-dimensional and Lipschitz continuous manifold;

b) There are uniform constants $\alpha>0, K>0$, and $\eta>0$, such that for any given bounded set $\Omega$ in $V$, there is a constant $t_{0}=t_{0}(\Omega)$, such that for any initial state $u_{0} \in \Omega$, the solution satisfies

$$
\operatorname{dist}_{V}(u(t), \mathcal{M}) \leq \alpha+K \exp \left(-\eta\left(t-t_{0}\right)\right) \text {, for } t \in\left[t_{0}, \infty\right) \text {. }
$$

c) For any trajectory $u(t)$ of the equation (1.1), there is a tracking orbit $v(t)$ (which may not be a trajectory) on the manifold $\mathcal{M}$, such that after a transient period $\left[0, t_{0}\right]$, the error satisfies the same exponential bound as above, i.e.

$$
\|u(t)-v(t)\| \leq \alpha+K \exp \left(-\eta\left(t-t_{0}\right)\right), \quad \text { for } t \in\left[t_{0}, \infty\right) .
$$

DEFINITION 5.2. A sequence of subsets $\left\{\mathcal{M}_{k}\right\}_{k=1}^{\infty}$ in $V$ is called a regular chain of approximate inertial manifolds if each $\mathcal{M}_{k}$ is an AIM in the above sense, $\operatorname{dim} \mathcal{M}_{k}$ is nondecreasing, and the thickness $\alpha\left(\mathcal{M}_{k}\right)$ decreases and converges to zero as $k \rightarrow \infty$.

The argument in proving the main theorem is the localizing and pasting technicality as follows. Let $\Delta>0$ be arbitrarily given. Denote by

$$
\begin{gathered}
t_{n}=t_{0}+n \Delta, \quad x_{n}=x\left(t_{n}\right)=P u\left(t_{n}\right), \quad z_{n}=z\left(t_{n}\right)=Q u\left(t_{n}\right), \quad h=Q f, \\
p_{n}=\left|z\left(t_{n}\right)-\varphi\left(x\left(t_{n}\right), Q f\right)\right|=\left|z_{n}-\varphi\left(x_{n}, Q f\right)\right|,
\end{gathered}
$$




$$
d_{n}=\left\|z\left(t_{n}\right)-\varphi\left(x\left(t_{n}\right), Q f\right)\right\|=\left\|z_{n}-\varphi\left(x_{n}, Q f\right)\right\| .
$$

Let $w_{n}(t):\left[t_{n}, t_{n+1}\right] \rightarrow Q V$ be the unique (classical) solution of the following equation with the initial value condition:

$$
\begin{gathered}
\underset{d t}{d} w_{n}+A_{Z} w_{n}+Q F\left(x_{n}+w_{n}(t)\right)=h, t \in\left[t_{n}, t_{n+1}\right], \\
w_{n}\left(t_{n}\right)=z_{n},
\end{gathered}
$$

LEMMA 5.3. Assume that $\mu>2\left(c_{2}\right)^{2}$. The above defined $\left\{p_{n}\right\}$ and $\left\{d_{n}\right\}$ satisfy

$$
\begin{aligned}
& p_{n} \leq \frac{\xi}{1-a}+a^{n} p_{o}, n \geq 1 \\
& d_{n} \leq \frac{\zeta}{1-b}+b^{n} d_{o}, n \geq 1
\end{aligned}
$$

where the constants are given by

$$
\begin{gathered}
a=\exp \left(-\left(\mu-c_{2} \mu^{1 / 2}\right) \Delta\right), \quad \xi=\frac{2 q c_{2}}{\mu-c_{2} \mu^{1 / 2}} \Delta^{1 / 2}, \\
b=\exp \left(-\frac{1}{2}\left(\mu-\left|c_{2}\right|^{2}\right) \Delta\right), \zeta=q c_{2}\left[\sqrt{\frac{2}{\mu-2\left(c_{2}\right)^{2}}}+\frac{1}{\mu^{1 / 2}-c_{2}} \Delta^{1 / 2} .\right.
\end{gathered}
$$

PROOF. By Lemma 4.1 we have

$$
\begin{aligned}
& \left|w_{n}(t)-\varphi\left(x_{n}, h\right)\right| \leq p_{n} \cup \operatorname{xp}\left(-\left(\mu-c_{2} \mu^{1 / 2}\right)\left(t-t_{n}\right)\right), \text { for } t \in\left[t_{n}, t_{n+1}\right], \\
& \left\|w_{n}(t)-\varphi\left(x_{n}, h\right)\right\| \leq d_{n} \exp \left(-\frac{1}{2}\left(\mu-\left|c_{2}\right|^{2}\right)\left(t-t_{n}\right)\right), \text { for } t \in\left[t_{n}, t_{n+1}\right] .
\end{aligned}
$$

We can estimate $p_{n+1}$ by

$$
\begin{gathered}
p_{n+1}=\left|z\left(t_{n+1}\right)-\varphi\left(x_{n+1}, h\right)\right| \\
s\left|z\left(t_{n+1}\right)-w_{n}\left(t_{n+1}\right)\right|+\left|w_{n}\left(t_{n+1}\right)-\varphi\left(x_{n}, h\right)\right|+\left|\varphi\left(x_{n}, h\right)-\varphi\left(x_{n+1}, h\right)\right|,
\end{gathered}
$$

in which we use (4.13) for the first part, (5.6) for the second part, and (3.12) and (4.11) for the third part, to obtain

$$
\begin{aligned}
p_{n+1} & \leq \frac{q c_{2}}{\mu-c_{2} \mu^{1 / 2}} \Delta^{1 / 2}+p_{n} \exp \left(-\left(\mu-c_{2} \mu^{1 / 2}\right) \Delta\right)+\frac{1}{\mu-c_{2} \mu^{1 / 2}} c_{2}\left\|x_{n}-x_{n+1}\right\| \\
& \leq \frac{q c_{2}}{\mu-c_{2} \mu^{1 / 2}} \Delta^{1 / 2}+p_{n} \exp \left(-\left(\mu-c_{2} \mu^{1 / 2}\right) \Delta\right)+\frac{1}{\mu-c_{2} \mu^{1 / 2}} q c_{2} \Delta^{1 / 2} \\
& \leq \frac{2 q c_{2}}{\mu-c_{2} \mu^{1 / 2}} \Delta^{1 / 2}+p_{n} \exp \left(-\left(\mu-c_{2} \mu^{1 / 2}\right) \Delta\right)=a p_{n}+\xi, n=0,1,2, \ldots
\end{aligned}
$$

Note that $0<a<1$. Hence (5.3) follows. Similarly, we can estimate $d_{n+1}$ by

$$
d_{n+1} \leq\left\|z\left(t_{n+1}\right)-w_{n}\left(t_{n+1}\right)\right\|+\left\|w_{n}\left(t_{n+1}\right)-\varphi\left(x_{n}, h\right)\right\|+\left\|\varphi\left(x_{n}, h\right)-\varphi\left(x_{n+1}, h\right)\right\|
$$




$$
\begin{gathered}
\leq \sqrt{\mu-2\left(c_{2}\right)^{2}} \mathrm{qc}_{2} \Delta^{1 / 2}+d_{n} \exp \left(-\frac{1}{2}\left(\mu-\left|c_{2}\right|^{2}\right) \Delta\right)+\frac{1}{\mu^{1 / 2}-c_{2}} \mathrm{qc}_{2} \Delta^{1 / 2} \\
=b d_{n}+\zeta, \quad n=0,1,2, \ldots,
\end{gathered}
$$

where we used (4.14) for the first part, (5.7) for the second part, and (3.13) and (4.11) for the third part. Note that $0<b<1$. Hence (5.4) follows. The proof is completed.

Now we state and prove the main result on the existence and properties of AIM.

THEOREM 5.4. Under the Assumptions (A1) and (A2), there exists an approximate inertial manifold $\mathcal{M}_{\mu}$ for the semiflow generatea $\because$ ' the evolution equation (1.1), which is given by

$$
\mathcal{M}_{\mu}=\operatorname{Graph} \varphi(., Q f)=\left\{\mathbf{x}+\varphi(x, Q f): \text { for all } \mathbf{x} \in \mathbf{X}_{\mu}\right\},
$$

where $\varphi: X_{\mu} \times Z_{\mu} \rightarrow Z_{\mu}$ is the dertermining mapping associated with $\mu>2\left(c_{2}\right)^{2}$. The dimension of the manifold $\mathcal{M}_{\mu}$ is

$$
\operatorname{dim} \mathcal{M}_{\mu}=\operatorname{dim} X_{\mu}=\mathbf{n}(\mu) .
$$

For any solution $u(t)$ with $u(0)=u_{0} \in V$, the following attraction properties hold,

$$
\begin{aligned}
& \operatorname{dist}_{H}\left(u(t), \mathcal{M}_{\mu}\right) \leq J(\mu)+\left|Q u\left(t_{0}\right)-\varphi\left(P u\left(t_{0}\right), Q f\right)\right| \exp \left(-\left(\mu-c_{2} \mu^{1 / 2}\right)\left(t-t_{0}\right)\right), \text { for } t \geq t_{0}, \\
& \operatorname{dist}_{V}\left(u(t), \mathcal{M}_{\mu}\right) \leq N(\mu)+\left\|Q u\left(t_{0}\right)-\varphi\left(P u\left(t_{0}\right), Q f\right)\right\| \exp \left(-\frac{1}{2}\left(\mu-\left|c_{2}\right|^{2}\right)\left(t-t_{o}\right)\right), \text { for } t \geq t_{o},
\end{aligned}
$$

where $t_{o}=t_{0}\left(u_{o}\right)$ is the final entering time of the trajectory $u(t)$ into the absorbing ball $B(0 ; \rho)$. Furthermore, the thickness $\mathrm{J}(\mu)$ in $\mathrm{H}$ and the thickness $\mathrm{N}(\mu)$ in $\mathrm{V}$ satisfy

$$
\begin{gathered}
\mathrm{J}\left(\mu,=\text { const } \frac{1}{\left(\mu-\mathrm{c}_{2} \mu^{1 / 2}\right)^{3 / 2}},\right. \\
\mathrm{N}(\mu)=\text { const }\left[\frac{1}{\left(\mu-2\left(c_{2}\right)^{2}\right)^{1 / 2}}+\frac{1}{\mu^{1 / 2}-c_{2}}\right] \frac{1}{\left(\mu-\left(c_{2}\right)^{2}\right)^{1 / 2}} \leq \text { const } \frac{1}{\left(\mu^{1 / 2}-c_{2}\right)^{2}} .
\end{gathered}
$$

PROOF. For $u(t)$ with $u(0)=u_{0}$, there must be an interval $\left[t_{n}, t_{n+1}\right)$ which contains $t$. Using (4.13), (5.6) plus (5.3), and (3.12) plus (4.11) for the following three parts respectively, we can get

$$
\begin{aligned}
& \operatorname{dist}_{H}\left(u(t), \mathcal{M}_{\mu}\right) \leq|[x(t)+z(t)]-[x(t)+\varphi(x(t), h)]|=|z(t)-\varphi(x(t), h)| \\
& \leq\left|z(t)-w_{n}(t)\right|+\left|w_{n}(t)-\varphi\left(x_{n}, h\right)\right|+\left|\varphi\left(x_{n}, h\right)-\varphi(x(t), h)\right| \\
& \leq \frac{q c_{2}}{\mu-c_{2} \mu^{1 / 2}} \Delta^{1 / 2}+\left(\frac{\xi}{1-a}+a^{n} p_{o}\right) \exp \left(-\left(\mu-c_{2} \mu^{1 / 2}\right)\left(t-t_{n}\right)\right)+\frac{q c_{2}}{\mu-c_{2} \mu^{1 / 2}} \Delta^{1 / 2} \\
& \leq \frac{2 q c_{2}}{\mu-c_{2} \mu^{1 / 2}}\left(1+\frac{1}{1-a}\right) \Delta^{1 / 2}+p_{0} \exp \left(-\left(\mu-c_{2} \mu^{1 / 2}\right)\left(t-t_{0}\right)\right), \text { for } t \geq t_{o},
\end{aligned}
$$

where in the last inequality (5.5) is used. venote by

$$
J(\mu)=\frac{2 q c_{2}}{\mu-c_{2} \mu^{1 / 2}}\left(1+\frac{1}{1-a}\right) \Delta^{1 / 2},
$$

where, let $\delta=\Delta^{1 / 2}$ and 


$$
\sigma(\delta)=\stackrel{1}{1-a^{1 / 2}=} \Delta_{1-\exp \left(-\left(\mu-c_{2} \mu^{1 / 2}\right) \delta^{2}\right)},
$$

then we can find the minimum value of $x(\delta)$ attained at $\delta^{*}>0$ such that $\omega^{*}=\left(\mu-c_{2} \mu^{1 / 2}\right) \delta^{* 2}$ satifsies the critical point equation

$$
\exp \left(\omega^{*}\right)=1+2 \omega^{*}, \text { so that } \omega^{*} \approx 1.26
$$

Hence we get

$$
\min \sigma(\delta)=\sigma\left(\delta^{*}=\frac{\sqrt{\omega^{*}}}{\left(\mu-c_{2} \mu^{1 / 2}\right)^{1 / 2}}\right)=\frac{\sqrt{\omega^{*}}}{1-\exp \left(-\omega^{*}\right)} \frac{1}{\left(\mu-c_{2} \mu^{1 / 2}\right)^{1 / 2}}
$$

Substitute this into (5.18) and (5.17), we obtain (5.13) and (5.15) in which the const $=2 q c_{2}\left(\omega^{*}\right)^{1 / 2}[1+(1$ $\left.\left.-\exp \left(-\omega^{*}\right)\right)^{-1}\right]$. Similarly, we have

$$
\begin{gathered}
\operatorname{dist}_{V}\left(u(t), M_{\mu}\right) \leq\|[x(t)+z(t)]-[x(t)+\varphi(x(t), h)]\|=\|z(t)-\varphi(x(t), h)\| \\
\leq\left\|z(t)-w_{n}(t)\right\|+\left\|w_{n}(t)-\varphi\left(x_{n}, h\right)\right\|+\left\|\varphi\left(x_{n}, h\right)-\varphi(x(t), h)\right\| \\
\leq \sqrt{\frac{2}{\mu-2\left(c_{2}\right)^{2}}} q c_{2} \Delta^{1 / 2}+\left(\frac{\zeta}{1-b}+b^{n} d_{0}\right) \exp \left(-\frac{1}{2}\left(\mu-\left|c_{2}\right|^{2}\right)\left(t-t_{n}\right)\right)+\frac{1}{\mu^{1 / 2}-c_{2}} q c_{2} \Delta^{1 / 2} \\
\leq\left[\sqrt{\frac{2}{\mu-2\left(c_{2}\right)^{2}}}+\frac{1}{\mu 1 / 2-c_{2}}\right]\left(1+\frac{1}{1-b}\right) q c_{2} \Delta^{1 / 2}+d_{o} \exp \left(-\frac{1}{2}\left(\mu-\left|c_{2}\right|^{2}\right)\left(t-t_{0}\right)\right),
\end{gathered}
$$

for $t \geq t_{0}$, where in the second inequality we used (4.14), (5.7) plus (5.4), and (3.13) plus (4.11) for the three parts respectively, and in the third inequality we used (5.5). Denote by

$$
N(\mu)=\left[\sqrt{\frac{2}{\mu-2\left(c_{2}\right)^{2}}}+\frac{1}{\mu^{1 / 2}-c_{2}}\right]\left(1+\frac{1}{1-b}\right) q c_{2} \Delta^{1 / 2},
$$

where, let $\delta=\Delta^{1 / 2}$ and

$$
\pi(\delta)=\frac{1}{1-b} \Delta^{1 / 2}=\frac{\delta}{1-\exp \left(-\frac{1}{2}\left(\mu-\left|c_{2}\right|^{2}\right) \delta^{2}\right)},
$$

then we can find the minimum value of $\pi(\delta)$ attained at

$$
S^{* *}=\left(\frac{2 \omega^{*}}{\mu-\left|c_{2}\right|^{2}}\right)^{1 / 2}
$$

where $\omega^{*}$ is the same as above. Hence we get

$$
\min \pi(\delta)=\pi\left(\delta^{* *}\right)=\frac{\sqrt{2 \omega^{*}}}{1-\exp \left(-\omega^{*}\right)} \frac{1}{\left(\mu-\left|c_{2}\right|^{2}\right)^{1 / 2}} .
$$

Substitute this into (5.20) and (5.19), then we obtain (5.14) and (5.16) in which the last const $=(1+\sqrt{2})$ $\mathrm{qc}_{2}\left(2 \omega^{*}\right)^{1 / 2}\left[1+\left(1-\exp \left(-\omega^{*}\right)\right)^{-1}\right]$. 
According to Definition 5.1, this set $\mathcal{M}_{\mu}$ given by (5.11) in $\mathrm{V}$ is an AIM for the semiflow generated by the evolution equation (1.1). We emphasize specifically the obtained attraction property: By (5.17) with (5.18) and (5.19) with (5.20), any solution $u(t)$ of (1.1), after its entering into the absorbing ball, will be tracked in the sense of (5.13) and (5.14) by an orbit $v(t)$ given below on the AIM $\mathcal{M}_{\mu}$,

$$
v(t):=P u(t)+\varphi(P u(t), Q f)
$$

Therefore the condition b) and condition c) in Definition 5.1 are both verified. The proof of Theorem 5.4 is completed.

COROLLARY 5.5. There is a regular chain of approximate inertial manifolds $\left\{\mathbf{M}_{\mathbf{k}}: \mathbf{k}=\left[2\left(\mathrm{c}_{2}\right)^{2}\right]+1\right.$, $\left.\left[2\left(c_{2}\right)^{2}\right]+2, \ldots\right\}$ for the semiflow generated by the evolution equation $(1.1)$.

PROOF. This is an immediate consequence of Definition 5.2 and the thickness formulas (5.18) and (5.20) for $\mu=$ intergers larger than $2\left(c_{2}\right)^{2}$. Therefore the assertion is true.

The obtained results can be generalized as follows. We replace the Assumption (A2) by the following Assumption (A3), and generalize the property (3.10) to the following Assumption (A4).

ASSUMPTION (A3). (dissipation condition) Assume that for any $u_{0} \in V$, there exists a unique global strong solution $u(t)$ of the equation (1.1) for $t \geq 0$ with $u(0)=u_{0}$. The semiflow in $V$ generated by this equation has the absorbing property. Moreover, after a truncation (3.3) holds.

ASSUMPTION (A4). (fractional monotonicity condition) Let $\mathrm{F}(\mathrm{u})$ be the truncated nonlinearity (3.1). Assume that there are constants $c_{3}>0$ and $0 \leq k<2$, such that

$$
\langle F(u)-F(v), u-v\rangle \geq-c_{3}\|u-v\|^{x}|u-v|^{2-x}, \text { for } u, v \in V .
$$

Now we state the correspondiny result under these generalized assumptions without proof which is parallel to the stream of our above treatment.

THEOREM 5.6. Under Assumptions (A1), (A3), and (A4), there exists an approximate inertial manifold $\mathcal{M}_{\mu}$, given by (5.11), for the semiflow generated by the evolution equation (1.1), with $\mu>$ $\max \left\{2\left(c_{2}\right)^{2},\left(c_{3}\right)^{2 /(2-k)}\right\}$. Moreover, it has the following attraction property,

$$
\operatorname{dist}_{V}\left(u(t), M_{\mu}\right) \leq \Gamma(\mu)+\left\|Q u\left(t_{0}\right)-\varphi\left(P u\left(t_{0}\right), Q f\right)\right\| \exp \left(-\frac{1}{2}\left(\mu-\left|c_{2}\right|^{2}\right)\left(t-t_{0}\right)\right), \text { for } t \geq t_{0}
$$

where $t_{o}=t_{0}\left(u_{o}\right)$ is the final entering time of the trajectory $u(t)$ into the absorbing set fixed in the truncatiom, and

$$
\Gamma(\mu) \approx \text { const } \frac{1}{\mu-c_{3} \mu^{2-\kappa}}, \text { as } \mu \rightarrow \infty .
$$

\section{APPLICATIONS}

In this section we present several application examples for which Theorem 5.4 or Theorem 5.7 can be used to assert the existence of a regular chain of approximate inertial manifolds.

BURGERS' EQUATION. As a simple model for various convection-diffusion phenomena and for turbulence flow, Burgers' equation has been studied for more than fifty years. Consider the following initial-boundary value problem of Burgers' equation:

$$
\begin{aligned}
\frac{\partial u}{\partial t}-v \frac{\partial^{2} u}{\partial x^{2}}+u \frac{\partial u}{\partial x} & =f(x),(t, x) \in R^{+} \times(0,1), \\
u(t, 0) & =u(t, 1)=0, t \geq 0, \\
u(0, x) & =u_{0}(x), \quad x \in[0,1] .
\end{aligned}
$$


Here $v>0$ is a constant. Let $H=L^{2}(0,1)$ with $t$ he norm denoted by $|$.$| and define an operator A$ : $D(A) \rightarrow H$ by $A=-v\left(d^{2} / d x^{2}\right)$ and $D(A)=H^{2}(0,1) \cap H_{0}^{1}(0,1)$. Then the operator A satisfies Assumption (A1). Let $V=D\left(A^{1 / 2}\right)=H_{0}^{1}(0,1)$ with the norm $\|v\|=\left|A^{1 / 2} v\right|$. Asume that $f \in V$ is constant in $t$. The nonlinearity is denoted by $R(u)=u u_{x}$.

The absorbing property and the existence of a global attractor are known. However, in the case $f \neq$ 0 , the attractor is nontrivial. On the other hand, the question of whether there exists an inertial manifold for (6.1) is still open in general, since the spectral gap condition is in the form

$$
\lambda_{n+1}-\lambda_{n} \geq K\left(\lambda_{n+1}^{1 / 2}-\lambda_{n}^{1 / 2}\right) \text { for a large } n .
$$

Note that $\lambda_{n}=2 \pi v n^{2}$, this condition reduces to $(2 \pi v)^{1 / 2} \geq K$, which is not valid in general. In Constantin et al. [16], the existence of inertial manifolds was proved for a modified nonlocal Burgers' equation with the nonlinear term $R(u)=\langle u, \omega\rangle u_{x}$ where $\omega \in H$ is given.

Here by verifying that the nonlinearity $R(u)=u u_{x}$ satisfies our Assumption (A2), we can assert that there is a regular chain of approximate inertial manifolds in the form of (5.11) for this Burgers' equstion. Note that $\mathrm{H}_{0}^{1}(0,1) \subset \mathrm{C}[0,1]$. We have:

$$
\begin{gathered}
|R(u)|=\left[\int_{0}^{1} u^{2}\left(u_{x}\right)^{2} d x\right]^{1 / 2} \leq(\sup \{|u(\xi)|: \xi \in[0,1]\})\|u\| \leq k\|u\|^{2} \text {, for } u \in V ; \\
\| R(u) !^{\prime \prime}=\left|(R(u))_{x}\right| \leq\left|\left(u_{x}\right)^{2}\right|+\left|u u_{x x}\right| \\
\leq\left|u_{x}\right|_{L^{4}}^{2}+\left(\sup \left\{|u(\xi)|^{2}: \xi \in[0,1]\right\}\right)|A u| \\
\leq C\left|u_{x}\right|\left|u_{x x}\right|+k^{2}\|u\|^{2}|A u|=\left(C\|u\|+k^{2}\|u\|^{2}\right)|A u|, \text { for } u \in D(A),
\end{gathered}
$$

where we used Gagliardo-Nirenberg inequality in getting $\left|u_{x}\right|_{L^{4}} \leq$ const $\left|u_{x}\right|^{1-\theta}\left|u_{x x}\right|^{\theta}$ with $-1 / 4 \leq \theta(1$ $1 / 2)-(1-\theta)(1 / 2)$ so that $\theta \geq 1 / 4$ and we take $\theta=1 / 2$, and $C$ is a constant.

$$
\begin{gathered}
|R(u)-R(v)| \leq\left(\int_{0}^{1} 2(u-v)^{2}\left(u_{x}\right)^{2} d x\right)^{1 / 2}+\left(\int_{0}^{1} 2 v^{2}\left(u_{x}-v_{x}\right)^{2} d x\right)^{1 / 2} \\
\leq 2 k\|u-v\|\|u\|+2 k\|v\|\|u-v\|=2 k(\|u\|+\|v\|)\|u-v\|, \text { for and } v \in V \\
\|R(u)-R(v)\| \leq\left|\left(u_{x}+v_{x}\right)\left(u_{x}-v_{x}\right)\right|+\left|(u-v) u_{x x}\right|+\left|v\left(u_{x x}-v_{x x}\right)\right| \\
\leq k(|A u|+|A v|)\|u-v\|+k\|u-v\||A u|+k\|v\||A u-A v| \\
\leq 2 k(|A u|+|i v|)|A u-A v|, \text { for } u \text { and } v \in D(A) ;
\end{gathered}
$$

and

$$
\langle R(u), u\rangle=\int_{0}^{1} u^{2} u_{x} d x=0 \geq-\beta\|u\|^{2}, \text { for } u \in V, \text { with any } 0<\beta<\lambda_{0}
$$

Therefore Assumption (A2) is fully satisfied. As a result, Theorem 5.4 can be applied to the semiflow generated by Burgers' equation (6.1), and the AIMs in the form of (5.11) with corresponding determining 
mapping exist and have the properties (5.12) through (5.14) for this semiflow.

REACTION-DIFFUSION EQUATIONS OF HIGH DIMENSION. Consider a reaction-diffusion equation

$$
\frac{d u}{d t}+A u+R(u)=f, t \geq 0
$$

where $A=-v \Delta u: D(A)\left(=H^{2}(\Omega) \cap H_{o}^{1}(\Omega)\right) \rightarrow H\left(=L^{2}(\Omega)\right)$, and $\Omega$ is an open, bounded and connected set of $R^{n}$ with a Lipschitz continuous boundary $\Gamma, f \in H_{0}^{1}(\Omega)$ is fixed, and $R(s)$ is a polynomial of odd degree with a positive leading coefficient, i.e.

$$
R(s)=\sum_{k=0}^{2 p-1} b_{k} s^{k}, p \geq 1 \text { (integer), } b_{2 p-1}>0 .
$$

In Temam [2], it was proved that there exists a global attractor with finite fractal dimension for this equation (6.7) and that for space dimension $n=1$ and 2 there exist inertial manifolds. In Mallet-Paret and Sell [17] the spatial averaging principle was applied to prove the existence of inertial manifolds for this type of equations with $n=3$ if $\Omega=(0.2 \pi)^{3}$. But for the higher dimensional case $(n \geq 3)$ with a general domain $\Omega$, the existence of inertial manifolds is unknown. Here we can apply our result of Theorem 5.4 to the higher dimensional reaction-diffusion equations with a general domain $\Omega$ by checking the conditions in (A2).

Denote by $V=H_{o}^{1}(\Omega)$ with the norm $\|v\|=\left(\sum_{i=1}^{n}\left|\partial v / \partial x_{i}\right|^{2}\right)^{1 / 2}$, where $|$.$| stands for the H-norm.$ Note that the absorbing property in $\mathrm{V}$ of (6.7) with (6.8) under the above conditions has been shown in Temam [2], we need only to verify the first conditions in 2 ), and the condition 3 ) of (A2).

We have, for any $u$ and $v \in V$,

$$
\begin{gathered}
|R(u)-R(v)|^{2} \leq 2 \sum_{k=1}^{2 p-1} \int_{\Omega}\left|b_{k}\right|^{2}\left[u(x)^{k}-v(x)^{k}\right]^{2} d x \\
\leq \text { const }\left[\int_{\Omega}|u(x)-v(x)|^{4} d x\right]^{1 / 2} \sum_{k=1}^{2 p-1}\left[\left(|u(x)|^{k-1}+|u(x)|^{k-2}|v(x)|+\ldots+\left.|v(x)|\right|^{k-1}\right)^{4} d x\right]^{1 / 2} \\
\leq \text { const }\|u-v\|^{2} \sum_{k=1}^{2 p-1}\left(\|u\|_{L^{2(k-1)}}^{2(k-1)}+\|v\|_{L^{2(k-1)}}^{2(k-1)}\right) \\
\leq \text { const }\|u-v\|^{2}\left(\|u\|_{L^{4(p-1)}}^{4(p-1)}+\|v\|_{L^{4(p-1)}}^{4(p-1)}\right) \\
\leq \text { const }\|u-v\|^{2}\left(\|u\|^{4(p-1)}+\|v\|^{4(p-1)}\right),
\end{gathered}
$$

where in the third inequality we used Gagliardo-Nirenberg inequality to get $\|u-v\|_{L^{4}} \leq$ const $\|u-v\|$, for $\mathrm{n} \leq 4$, and used Young's inequality to get $|\mathrm{u}(\mathrm{x})|^{2(\mathrm{k}-\mathrm{i})}|\mathbf{v}(\mathrm{x})|^{2 \mathrm{i}} \leq|\mathrm{u}(\mathrm{x})|^{2(\mathrm{k}-1)}+\mathrm{C}|\mathrm{v}(\mathrm{x})|^{2(\mathrm{k}-1)}$ with a constant $\mathrm{C}$ $=(p-1) / p^{q}$ and $p=(k-1) /(k-i), q=(k-1) / i, i=1, \ldots, k-2$, and then Holder inequality and Sobolev imbedding $L^{4(p-1)}(\Omega) \subset V$ ( valid if $1-n / 2 \geq-n /(4 p-4)$ ) are used respectively in getting the last two inequalities. 
Next we check the condition 3> of (A2). Note that by Young's inequality there is a uniform constant $D_{1}>0$ such that

$$
\sum_{k=0}^{2 p-2}\left|b_{k} s^{k+1}\right| \leq \frac{1}{2} b_{2 p-1}|s| 2 p+D_{1}, \text { for any } s \in R .
$$

Hence the condition 3) of (A2) is satisfied:

$$
\langle R(u), u\rangle \geq \int_{\Omega} \frac{1}{2} b_{2 p-1}|u(x)|^{2 p} d x-D_{1}|\Omega| \geq-D_{1}|\Omega| \text {, for any } u \in V .
$$

As a result, we can apply Theorem 5.4 to the reaction-diffusion equation (6.7) with (6.8), if

$$
n \leq 4 \text { and } \frac{2 p-3}{4 p-4} \leq \frac{1}{n}
$$

The second inequality is obtained from the only requirement inequality for the imbedding $L^{4(p-1)}(\Omega) \subset V$. Thus we can conclude that for the equation (6.7) with

$$
n=3 \text { or } n=4 \text {, and } 2 p-1=3 \text { (polynomial }(6.8) \text { of degree } 3 \text { with } b_{3}>0 \text { ), }
$$

there exist the AIMs in the form of (5.11) and have the properties (5.12) through (5.14).

2D GINZBURG-LANDAU EQUATIONS. The complex Ginzburg- Landau equation (with the Dirichlet boundary condition) in the following form:

$$
\begin{gathered}
\frac{\partial u}{\partial t}-(1+i v) \Delta u+(k+i \eta)|u|^{2} u-r u=0, \text { for }(t, x) \in R^{+} \times \Omega, \\
\left.u\right|_{\Gamma}=0, \text { for } t \geq 0,
\end{gathered}
$$

where $v, \eta$, and $\kappa$ are real numbers with $\kappa>0$, and $\Omega$ is an open, bounded, and connected set of $R^{n}$ ( $n \geq$ 1), with a boundary $\Gamma$ being Lipschitz continuous, can serve as mathematical models for the behavior of superconductors in a magnetic field and near the critical temperature, cf. Gorkov [18], nonlinear instable waves in plane Poiseuille flows, cf. Stewartson and Stuart [19], and other applications, cf. Temam [2]. Note that the existence of global atractor for $n \leq 2$ and the existence of inertial manifolds only for $n=1$ have been proved in Doering et al. [20]. However, for the space dimension $\mathbf{n}=\mathbf{2}$ and the general domain $\Omega$, the existence of inertial manifolds is an open issue.

Take the viewpoint to visualize a complex function as a vector of two real components, namely $u=$ $\operatorname{col}\left(u_{1}, u_{2}\right)$, set up $H=\left[L^{2}(\Omega)\right]^{2}$ with the norm $||,. V=\left[H_{o}^{1}(\Omega)\right]^{2}$ with the norm $\|$.$\| , define an operator$

$$
A=\left(\begin{array}{cc}
-\Delta v \Delta \\
-v \Delta-\Delta
\end{array}\right): D(A) \rightarrow H \text { with } D(A)=\left[H^{2}(\Omega) \cap H_{o}^{1}(\Omega)\right]^{2},
$$

and a nonlinear mapping

$$
B(u)=\left\{\begin{array}{l}
|u|^{2}\left(k u_{1}-\eta u_{2}\right) \\
\left.u\right|^{2}\left(\eta u_{1}+k u_{2}\right)
\end{array}\right.
$$

Then (6.13) can be expressed as the following evolution equations,

$$
\frac{d u}{d t}+A u+B(u)-r u=0, t \geq 0 .
$$


It is easy to verify that $A: D(A) \rightarrow H$ satisfies $(A 1)$. Denote by $R(u)=B(u)-r u$. Let $n=2$. Then one can verify that $R(u)$ satisfies (A2). Since the absorbing property was proved, cf. Temam [2], below we only check the third inequality condition and the fifth inequality condition of (A2).

For any $u$ and $v \in V$, we have

$$
\begin{gathered}
|\mathrm{R}(\mathrm{u})-\mathrm{R}(\mathrm{v})|^{2} \leq 2|\mathrm{~B}(\mathrm{u})-\mathrm{B}(\mathrm{v})|^{2}+2|\mathrm{r}|^{2}|\mathrm{u}-\mathrm{v}|^{2} \\
\leq \mathrm{const}\left(\mathrm{k}^{2}+\eta^{2}\right) \int_{\Omega}|\mathrm{u}(\mathrm{x})-\mathrm{v}(\mathrm{x})|^{2}\left[|\mathrm{u}(\mathrm{x})|^{2}+|\mathrm{v}(\mathrm{x})|^{2}\right] \mathrm{dx}+\mathrm{const}\|\mathrm{u}-\mathrm{v}\|^{2} \\
\leq \text { const }\left(\int_{\Omega}|\mathrm{u}(\mathrm{x})-\mathrm{v}(\mathrm{x})|^{4} \mathrm{~d} \mathbf{x}\right)^{1 / 2}\left(\int_{\Omega}\left[|\mathrm{u}(\mathrm{x})|^{4}+|\mathrm{v}(\mathrm{x})|^{4}\right] \mathrm{dx}\right)^{1 / 2}+\text { const }\|\mathrm{u}-\mathrm{v}\|^{2} \\
\leq \text { (by Sobolev embedding) const }\|\mathrm{u}-\mathrm{v}\|^{2}\left[\left(\|\mathrm{u}\|^{4}+\|\mathrm{v}\|^{4}\right)^{1 / 2}+1\right],
\end{gathered}
$$

and, for any $u \in V$,

$$
\begin{aligned}
\langle R(u), u\rangle & =\langle B(u), u\rangle-r|u|^{2}=\int_{\Omega}\left[k|u(x)|^{4}-r|u(x)|^{2}\right] d x \\
& =\kappa \int_{\Omega}\left[|u(x)|^{2}-\frac{r}{2 \kappa}\right]^{2} d x-\frac{r^{2}}{4 k} \geq-\frac{r^{2}}{4 \kappa} .
\end{aligned}
$$

Therefore the two conditions of concern are satisfied. We can apply Theorem 5.4 to the 2D GinzburgLandau equation (6.13) or (6.14) to assert that there exist the AIMs in the form of (5.11) and have the properties (5.12) through (5.14).

AXIALLY SYMMETRIC KURAMOTO-SIVASHINSKI EQUATIONS. In Foias et al. [21, 22], the existence of inertial manifolds in $\mathrm{H}^{1}$ Sobolev space for the Kuramoto-Sivashinsky equation of one space dimension was proved.The approximate inertial manifolds for the 1D Kuramoto-Sivashinsky equations with periodic boundary conditi ns constructed by several schemes including the concrete steadystate determining mapping has been presented in Jolly, Keverkidis, and Titi [12] based on the methods in Titi [11]. However, for the Kuramoto-Sivashinsky equation on 2D domains :

$$
u_{t}+\Delta^{2} u+\Delta u+\frac{1}{2}|\nabla u|^{2}=0, t \geq 0, x \in \Omega \subset R^{2},
$$

the theory of global dynamics is under exploration, since the dissipation has not been proved yet in general. Recently, Sell and Taboada [23] dealt with thin rectangular domains and periodic boundary conditions, and proved the local dissipativity and the existence of local attractors in $D\left(A^{1 / 4}\right)$.

Here we want to apply the result in this work to the axially symmetric Kuramoto-Sivashinsky equation on annular domains in $R^{2}$. Specifically, let $\Omega=\left\{(r, \theta): r_{0} \leq r \leq r_{1}\right.$ and $\left.0 \leq \theta<2 \pi\right\}$ with $r_{1}>r_{0}$ $>0$. The $2 \mathrm{D} \mathrm{K}$-S equation (6.17) on such a domain $\Omega$ with the Dirichlet boundary condition and in polar coordinates $(r, \theta)$ is given by

$$
\begin{gathered}
\frac{\partial u}{\partial t}+\frac{\partial^{4} u}{\partial r^{4}}+\frac{2}{r} \frac{\partial^{3} u}{\partial r^{3}}+\left(1+\frac{1}{r^{2}}\right) \frac{\partial^{2} u}{\partial r^{2}}+\frac{1}{r} \frac{\partial u}{\partial r}+\frac{1}{2}\left|\frac{\partial u}{\partial r}\right|^{2}=0, \text { for } t \geq 0,(r, \theta) \in \Omega, \\
\left.u\left(t, r_{o}, \theta\right)=u^{\prime} t, r_{1}, \theta\right)=0, \quad \text { for } t \geq 0, \theta \in[0,2 \pi) .
\end{gathered}
$$


Here we consider only the axially symmetric solutions of (6.18). Thus the equivalent equation and the boundary conditions of concern are given by

$$
\begin{gathered}
r \frac{\partial u}{\partial t}+r \frac{\partial^{4} u}{\partial r^{4}}+2 \frac{\partial^{3} u}{\partial r^{3}}+\left(r+\frac{1}{r}\right) \frac{\partial^{2} u}{\partial r^{2}}+\frac{\partial u}{\partial r}+\frac{r}{2}\left|\frac{\partial u}{\partial r}\right|^{2}=0, \text { for } t \geq 0, r \in\left[r_{0}, r_{1}\right], \\
u\left(t, r_{0}\right)=u\left(t, r_{1}\right)=0, \text { for } t \geq 0, \\
u(0, r)=u_{0}(r) \in V, \text { for } r \in\left[r_{0}, r_{1}\right] .
\end{gathered}
$$

Let $I=\left(r_{0}, r_{1}\right)$. Define Hilbert spaces $H=L^{2}(I)$ with the norm denoted by $|u|$ and $V=H^{2}(I) \cap H_{0}^{1}(I)$ with the norm $\|u\|=\left(\int\left|u_{m}(x)\right|^{2} d x\right)^{1 / 2}$. Define two linear operators $L u=r u_{t}$, and

$$
R(u)=\left(r+r^{-1}\right) u_{r r}+(r / 2)\left(u_{r}\right)^{2}: D\left(A^{1 / 2}\right)=V \rightarrow H
$$

Then (6.19) can be expressed by the following evolution equation:

$$
L u+A u+R(u)=0, \quad t \geq 0, u(0)=u_{0} \in V
$$

We first verify that the equation (6.20) possesses the absorbing property in $\mathrm{V}$, and then check that the crucial third inequality condition of (A2). For the first issue, take the inner-products of the both sides (6.19) with $-r^{-1} u_{r r}$, we have

$$
\begin{gathered}
\frac{1}{2} \frac{d}{d t}\left|u_{r}\right|^{2}+\left|u_{r r r}\right|^{2}-\left\langle\frac{2}{r} u_{r r r}, u_{r r}\right\rangle-\left\langle\left(1+\frac{1}{r^{2}}\right) u_{r r}, u_{r r}\right\rangle-\left\langle\frac{1}{r} u_{r}, u_{r r}\right\rangle-\frac{1}{2}\left\langle\left(u_{r}\right)^{2}, u_{r r}\right\rangle \\
=\frac{1}{2} \frac{d}{d t}\left|u_{r}\right|^{2}+\left|u_{r r}\right|^{2}-\left\langle\left(1+\frac{2}{r^{2}}\right) u_{r r}, u_{r r}\right\rangle-\left\langle\frac{1}{r^{2}} u_{r}, u_{r}\right\rangle=0 .
\end{gathered}
$$

By Young's inequality, we have constar... $C_{1}\left(\varepsilon_{1}\right)>0$ and $C_{2}\left(\varepsilon_{2}\right)>0$, which depend on small $\varepsilon_{1}>0$ and $\varepsilon_{2}>0$ respectively, such that

$$
\begin{gathered}
\left|\left\langle\left(1+\frac{2}{r^{2}}\right) u_{r r}, u_{r r}\right\rangle\right| \leq \varepsilon_{1}\left\|u_{r r}\right\|_{L^{4}}^{2}+C\left(\varepsilon_{1}\right) \leq \frac{1}{4}\left|u_{r m}\right|^{2}+C_{1}, \\
\left|\left\langle\frac{1}{r^{2}} u_{r}, u_{r}\right\rangle\right| \leq \varepsilon_{2}\left\|u_{r}\right\|_{L^{4}}^{2}+C\left(\varepsilon_{2}\right) \leq \frac{1}{4}\left|u_{r r r}\right|^{2}+C_{2},
\end{gathered}
$$

where $C_{1}$ and $C_{2}>0$ are constants. Substitute (6.22) into (6.21), we get

$$
\frac{d}{d t}\left|u_{r}\right|^{2}+\left|u_{r m}\right|^{2} \leq 2\left[C_{1}+C_{2}\right]
$$

which implies that the strong solution of (6.19) in $H^{1}(I)$ exists globally and there exists an absorbing set in $H^{1}(I)$. Next take the inner-products of the both sides (6.19) with $r^{-1} u_{r r r}$ to get

$$
\begin{aligned}
& \frac{1}{2} \frac{d}{d t}\left|u_{m r}\right|^{2}+\left|u_{m m}\right|^{2}+\left\langle\frac{2}{r} u_{m r}, u_{m m}\right\rangle+\left\langle\left(1+\frac{1}{r^{2}}\right) u_{r r}, u_{r m r}\right\rangle+\left\langle\frac{1}{r} u_{r}, u_{r m r}\right\rangle+\frac{1}{2}\left\langle\left(u_{r}\right)^{2}, u_{r m r}\right\rangle
\end{aligned}
$$

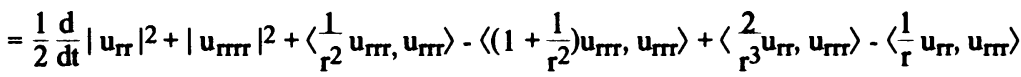

$$
\begin{aligned}
& +\left\langle\frac{1}{r^{2}} u_{r}, u_{m r}\right\rangle-\left\langle u_{r} u_{m r}, u_{m r}\right\rangle
\end{aligned}
$$




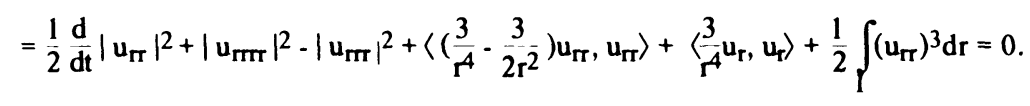

Similarly we have

$$
\begin{gathered}
\left|u_{r \pi}\right|^{2} \leq \frac{1}{6}\left|u_{r r \pi}\right|^{2}+C_{3}\left|u_{r}\right|^{2}, \\
\left|\left\langle\frac{3}{2 r^{2}} u_{r r}, u_{r r}\right\rangle\right| \leq \varepsilon_{3}\left\|u_{r r}\right\|_{L 4}^{2}+C\left(\varepsilon_{3}\right) \leq \frac{1}{6}\left|u_{r r r}\right|^{2}+C_{4}, \\
\left|\frac{1}{2} \int\left(u_{r r}\right)^{3} d r\right|=\frac{1}{2}\left\|u_{\pi r}\right\|_{L^{3}}^{3} \leq \text { const }\left|u_{r r r}\right|^{7 / 4}\left|u_{r}\right|^{5 / 4} \leq \frac{1}{6}\left|u_{r r m}\right|^{2}+C_{5}\left|u_{r}\right|^{10},
\end{gathered}
$$

where $C_{3}, C_{4}$, and $C_{5}>0$ are constants. Substitute (6.25) into (6.24), we obtain

$$
\frac{d}{d t}\left|u_{\pi r}\right|^{2}+\left|u_{m \pi}\right|^{2} \leq C_{3}\left|u_{r}\right|^{2}+C_{4}+C_{5}\left|u_{r}\right|^{10}
$$

This, together with the fact that $\left|u_{r}\right|^{2} \leq\left|u_{o r}\right|^{2} \exp (-v t)+2\left(C_{1}+C_{2}\right) v v$ for some constant $v>0$ from the previous step (6.23), implies that the strong solution of (6.19) in $\mathrm{H}^{2}(\mathrm{I})$ exists globally and there exists an absorbing set in $\mathrm{H}^{2}(\mathrm{I})$. Thus it remains to verify the crucial first condition of 2 ) in (A2) as follows: for any $\mathrm{u}$ and $\mathbf{v}$ in $\mathrm{H}^{2}(\mathrm{I})$,

$$
\begin{gathered}
|R(u)-R(v)|^{2} \leq 2\left|\left(r+r^{-1}\right)\left(u_{\pi}-v_{r r}\right)\right|^{2}+2\left|(r / 2)\left(u_{r}-v_{r}\right)\left(u_{r}+v_{r}\right)\right|^{2} \\
\leq \text { const }\|u-v\|^{2}+\text { const }\left\|u_{r}-v_{r}\right\|_{L^{4}}^{2}\left(\left\|u_{r}\right\|_{L^{4}}^{2}+\left\|v_{r}\right\|_{L^{4}}^{2}\right) \\
\leq \text { const }\|u-v\|^{2}\left(1+\|u\|^{2}+\|v\|^{2}\right) .
\end{gathered}
$$

Therefore, We can apply Theorem 5.4 to the axially symmetric Kuramoto-Sivashinsky equation (6.19) to assert that there exist the AIMs in the form of (5.11) and have the properties (5.12) through (5.14).

\section{REFERENCES}

1. HALE, J.K. Asymptotic Behavior of Dissipative Systems, Math Survey \& Monographs, No. 25, AMS, Providence, RI, 1988.

2. TEMAM, R. Infinite Dimensional Dynamical Systems in Physics and Mechanics, Springer-Verlag, New York, 1988.

3. TABOADA, M. and YOU, Y. Global attaractor, inertial manifolds and stabilization of nonlinear damped beam equations, (1992), to appear in Comm. Partial Diff. Eqns.

4. TEMAM, R. Induced trajectories and approximate inertial manifolds, Math Modelling and Numer. Anal., 23 (1989), 541-561.

5. FOIAS, C. et al. Exponential tracking and approximation of inertial manifolds for dissipative equation, Journal of Dynamics and Diff. Eqns., 1 (1989), 199-224.

6. MARION, M. Approximate inertial manifolds for reaction-diffusion equations in high space dimension, Joumal of Dynamics and Diff. Eqns., 1 (1989), 245-267.

7. SELL, G.R. Approximation dynamics: hyperbolic sets and inertial manifolds, IMA Preprint, 1989.

8. FOIAS, $C$. et al. Sur l'interaction des petits et grands tourbillans dans les ecoulements turbulents, $\underline{C}$. R. Acad. Sci. Paris, Ser. I, 305 (1987), 497-500.

9. FOIAS, C. et al.. Modelization of the interaction of small and large eddies in two dimensional turbulent flows, Math. Modelling \& Numer. Anal., 22 (1988), 93-114. 
10. TITI E.S. Une Variete approximate 'e l'attracteur universel des equations de Navier-Stokes, nonlineaire, de dimension finie, C.R. Acad. Sci. Paris, Ser. I, 307 (1988), 383-385.

11. TITI, E.S. On approximate inertial manifolds to the Navier-Stokes equations, J. Math. Anal. Appl., 149 (1990), 540-557.

12. JOLLY, M, et al. Approximate inertial manifolds for Kuramoto-Sivashinski equation: analysis and computations, Physica D, 44 (1990), 38-60.

13. SMILEY, M. Global attractors and approximate inertial manifolds for abstract dissipative equations, IMA Preprint, 1990.

14. PAZY, A. Semigroups of Linear Operators and Applications to Partial Differential Equations, Springer -Verlag, New York, 1983.

15. CHOW, S.N. and HALE, J.K. Methods of Bifurcation Theory, Springer-Verlag, New York, 1982.

16. CONSTANTIN, P. et al. Integral Manifolds and Inertial Manifolds for Dissipative Partial Differential Equations,Springer-Verlag, New York, 1988.

17. MALLET-PARET, J. and SELL, G.R. Inertial manifolds for reaction-diffusion equations in higher space dimensions, Joumal of Amer. Math. Soc., 1 (1988), 805-866.

18. GORKOV, L.P. Microscopic derivation of the Ginzburg-Landau equations in the theory of superconductivity, Soviet Physics J.E.T.P., 36 (1959), 1364- 1367.

19. STEWARDSON, K. and STIART J.T. A nonlinear instability theory for a wavw system in plane Poiseuille flow, J. Fluid Mech., 48 (1971), 529-545.

20. DOERING, C. et al. Low-dimensional behavior in the complex Ginzburg-Landau equations, Nonlinearity, 1(1988), 279-309.

21. FOIAS, C. et al. Varietes inertielles pour l'equation de Kuramoto-Sivashinsky, C. R. Acad. Sc. Paris, Ser. I, 301 (1985), 285-288.

22. FOIAS, C. et al. Inertial manifolds for the Kuramoto-Sivashinski equation and an estimate of their lowest dimension, J. Math. Pures Appl., 67 (1988), 197-226.

23. SELL, G.R. and TABOADA, M. Local dissipativity and attractors for the Kuramoto -Sivashinski equation in thin 2D domains, IMA Preprint, 1991. 


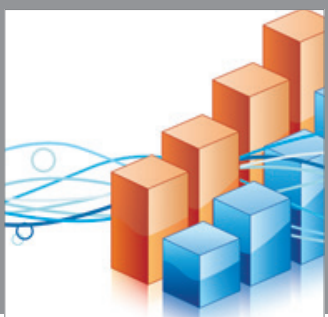

Advances in

Operations Research

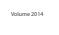

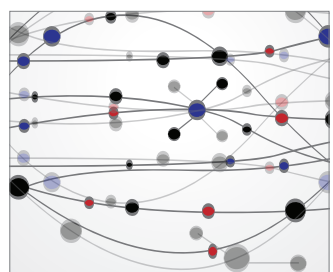

\section{The Scientific} World Journal
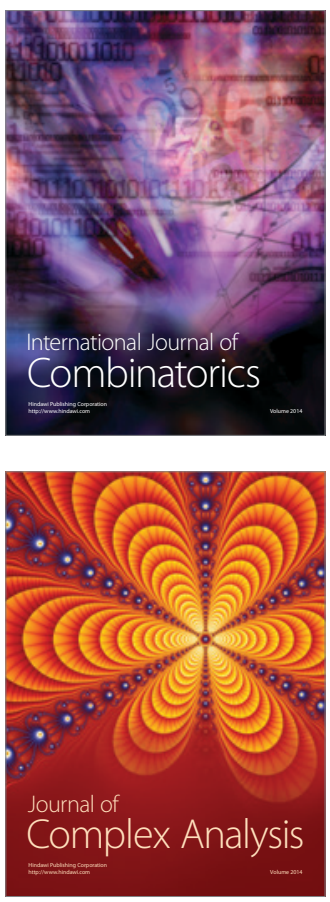

International Journal of

Mathematics and

Mathematical

Sciences
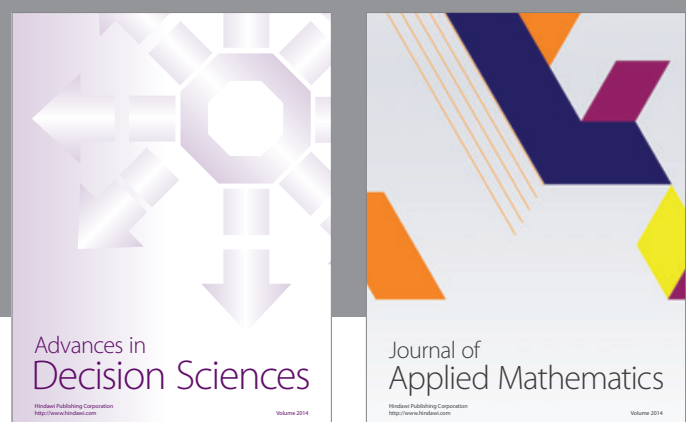

Journal of

Applied Mathematics
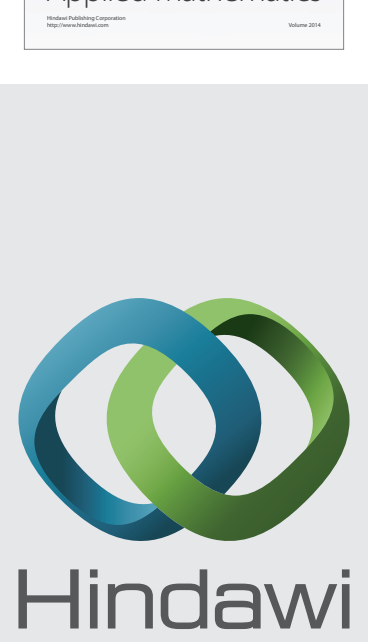

Submit your manuscripts at http://www.hindawi.com
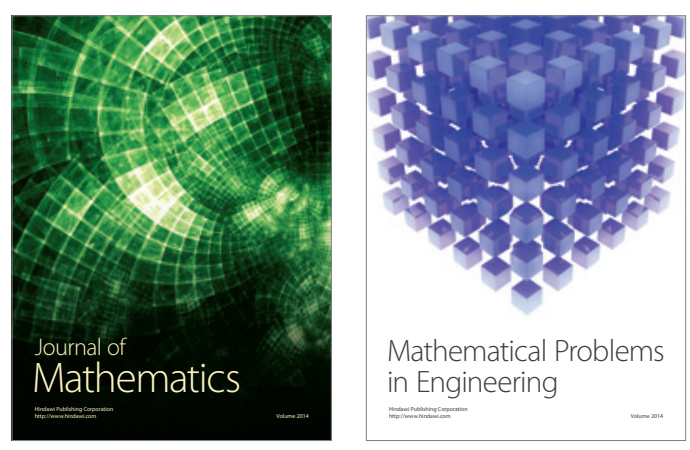

Mathematical Problems in Engineering
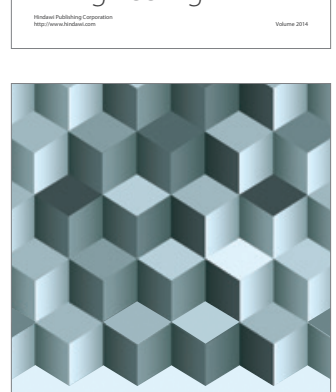

Journal of

Function Spaces
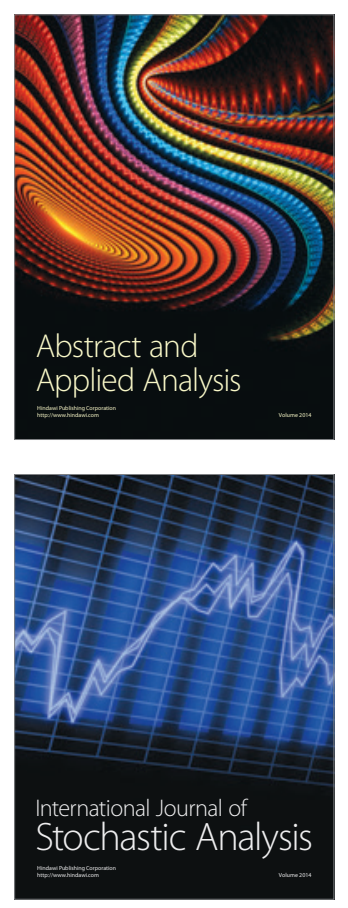

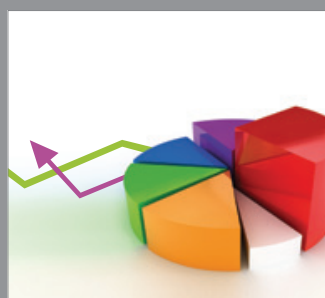

ournal of

Probability and Statistics

Promensencen
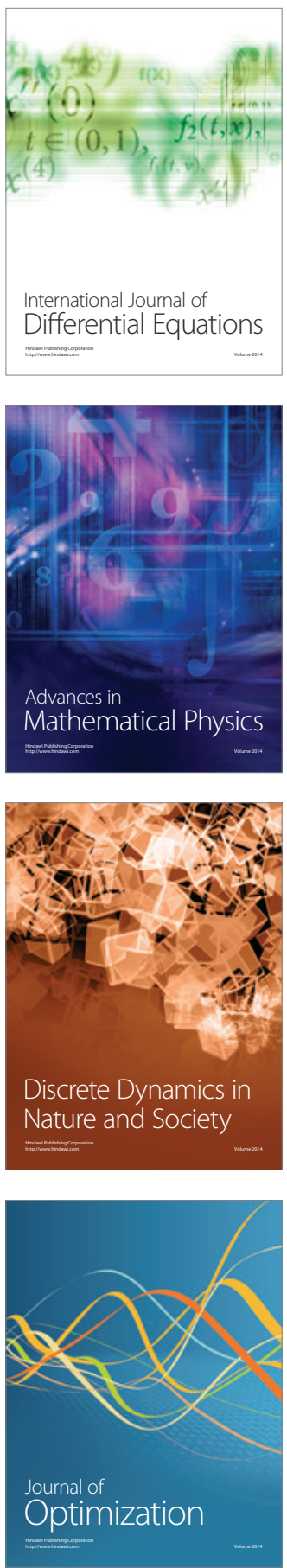